Research Article

\title{
Signal Detection from Permutated Observations Using Distributed Sensors
}

\author{
Naiti Jiang ${ }^{\circledR},{ }^{1,2}$ Ning Zhang ${ }^{\circledR},{ }^{2,3}$ and Jindong Zhang ${ }^{4}$ \\ ${ }^{1}$ Nanjing University of Information Science and Technology, China \\ ${ }^{2}$ Nanjing Marine Radar Institute, China \\ ${ }^{3}$ Tsinghua University, China \\ ${ }^{4}$ Nanjing University of Aeronautics and Astronautics, China \\ Correspondence should be addressed to Naiti Jiang; jiangnt_ee@163.com
}

Received 29 December 2018; Accepted 20 February 2019; Published 7 August 2019

Academic Editor: Antonio Lazaro

Copyright (c) 2019 Naiti Jiang et al. This is an open access article distributed under the Creative Commons Attribution License, which permits unrestricted use, distribution, and reproduction in any medium, provided the original work is properly cited.

In this paper, distributed constant level detection in wireless sensor networks (WSNs) is investigated. The permuted linear model with a scalar parameter and additive heteroscedastic Gaussian noise is introduced, where the associations between the observations and the sensors are assumed to be unknown. Several detectors such as the approximations of the generalized likelihood ratio test (GLRT) detector, mean detector, and energy detector are proposed, and their receiver operating characteristics (ROCs) are evaluated. Numerical simulations are performed, and it is shown that the performance degradation of the GLRT detector is small, compared to the permutation known as Neyman-Pearson (NP) detector.

\section{Introduction}

Decentralized estimation and detection in wireless sensor networks (WSNs) has attracted considerable attention in the past decades [1-11]. Early in [1], an overall description of decentralized detection was presented. Years later, a two-part review summed up the basic results of distributed detection more detailedly $[2,3]$. In part $I$, the authors reviewed the fundamentals and discussed several important issues, including the computational complexity, network design with general topologies, and applications in different areas. In part II, further works on new ideas of asymptotically optimum, nonparametric, robust, and sequential centralized detection are investigated. Advancements in these directions provide more efficient detection schemes by optimizing more general performance criteria.

In general, observations collected from WSNs can be mathematically expressed as a linear noisy model. The most common assumption in existing literature is that the observation matrix is perfectly known and the observations are perfectly labeled. Then the unknown signal can be detected or directly recovered from the observations by the least squares methods. One variant of the scenario is that there exists model uncertainty where multiplicative noise is introduced $[7,8,12-15]$. A more complex scenario corresponds to the case that the observation coefficients are partially known, which is also known as unlabeled sensing [16-22]. In this setup, the detection problem from unlabeled data is closely related to the problem of parameter estimation. To obtain the generalized likelihood ratio test (GLRT), usually one has to estimate both the permutation matrix and the unknown parameters via numerical algorithms. In [16], a branch and bound global optimization algorithm combined with the signal's sparsity information is utilized, and numerical results demonstrate that permutation matrix can be recovered correctly under certain circumstances. In [17], it is shown that the underlying signal can be recovered correctly with probability one, given that the sensing matrix is a random matrix with i.i.d. entries and the number of measurements is twice as many as that of unknowns. Taking the observation noises into account, it has been shown that recovery of the permutation matrix depends sharply on the signal-to-noise ratio, the number of measurements, and the estimated parameters [18]. One important variant of the above problem is the unlabeled ordered sampling problem, i.e., only a subset of measurements is kept and the relative 
order is preserved, an alternating minimization algorithm is proposed, and phase transition phenomenon was revealed in [19]. In [20], the permutated linear regression problem with a scalar parameter and additive heteroscedastic Gaussian noise is studied, and an alternating minimization algorithm is proposed to jointly recover the permutation matrix and the underlying parameter. In [23], signal amplitude estimation and detection from unlabeled binary samples are studied, and the number of quantizers needed to recover the permutation matrix is provided. It is noteworthy that the unlabeled sensing problems appear on many applications such as archaeological measurements, time jitter in sampling, and multitarget tracking [24-26].

In this paper, the problem of distributed constant level detection from unlabeled network observations is studied. Technically, it would be a tough task to accurately associate enormous observations generated by heterogeneous sensors in the big data era, which directly motivates the present work. More explicitly, we consider the case that the association between the sensors and the observed data is unknown while the time association is accurate. The goal is to detect whether the desired scalar signal is present or absent based on the permutated data. Compared to the deterministic detection problem discussed in [27], we are faced with a unknown signal contaminated by additive noises. More importantly, the detection problem is considered in a sensing communication model, where each sensor transmits the unlabeled noisy data to the fusion center (FC) for decision making. While theoretical analysis for parameter estimation under the same model has been published in [20], unknown scalar detection is still an open problem to be addressed. We fill this lack in this work and main contribution of the presented paper is the valuable solutions to the detection problem as well as the proposed methods to improve the detection performance.

\section{Problem Setup}

The system model is described in this section, under which the ML estimation with reference to the observations and the clairvoyant detector are derived. As mentioned earlier, we study the detection problem from permuted data in WSNs. Considering such a detection scenario, there are $N$ sensors collecting the data of a noisy scalar parameter $\theta$. Given that $i$ and $j$ denote the sensor and the time index, respectively, we suppose that the randomness in the unknown scalar signal $\mathbf{X}$ can be summarized in the following binary hypothesis testing problem:

$$
\left\{\begin{array}{l}
\mathscr{H}_{0}: X_{i j}=W_{i j}, i=1, \cdots, N, \\
\mathscr{H}_{1}: X_{i j}=\theta+W_{i j}, j=1, \cdots, K
\end{array}\right.
$$

where $X \in R^{N \times K}$ is the unknown scalar signal and $W_{i j} \sim$ $N\left(0, \sigma_{w}^{2}\right)$. Then all sensors transmit the noisy data to the fusion center (FC) through a channel. Here, we suppose that the channel between the $i$ th sensor and the FC is timeinvariant, and the channel coefficient is denoted by $h_{i}$. Then the observations after the transmission $Y_{i j}$ can be expressed as follows:

$$
Y_{i j}=h_{i} X_{i j}+v_{i j}
$$

where $v_{i j}$ is an i.i.d. noise sequence satisfying $v_{i j} \sim N\left(0, \sigma_{v}^{2}\right)$ and is independent of $W_{i j}$.

Assume that FC only knows that $Y_{i j}$ belongs to one sensor, i.e., the time association is accurate and imposes a preprocess procedure on the raw data as follows:

$$
y_{i}=\frac{\sum_{j=1}^{K} Y_{i j}}{K} .
$$

Under hypothesis $\mathscr{H}_{1}$, equation (3) can be simplified as follows:

$$
y_{i}=\frac{\sum_{j=1}^{K}\left(h_{i} X_{i j}+v_{i j}\right)}{K}=\frac{\sum_{j=1}^{K}\left(h_{i} \theta+h_{i} W_{i j}+v_{i j}\right)}{K}=h_{i} \theta+e_{i},
$$

where $\mathbf{e}$ is defined as follows:

$$
e_{i} \triangleq \frac{\sum_{j=1}^{K}\left(h_{i} W_{i j}+v_{i j}\right)}{K}
$$

Let $\mathbf{e}=\left[e_{1}, \cdots, e_{N}\right]^{\mathrm{T}}$. It follows that $e \sim N(0, W)$, where $\mathbf{W}$ is a diagonal matrix whose diagonal element is $\left(h_{i}^{2} \sigma_{w}^{2}+\sigma_{v}^{2}\right) / K$. Since data $\left\{y_{i}\right\}_{i=1}^{N}$ is unlabeled and it follows that

$$
\bar{y}=\Pi(h \theta+e),
$$

where $\bar{y}$ is the unlabeled observations of $\mathbf{y}$, and $\boldsymbol{\Pi}$ is an unknown $N \times N$ permutation matrix which disorders the rows of the labeled observations. As a consequence, the detection problem can be formulated as follows:

$$
\left\{\begin{array}{l}
\mathscr{H}_{0}: \bar{y}=\Pi e \\
\mathscr{H}_{1}: \bar{y}=\Pi(h \theta+e) .
\end{array}\right.
$$

It is noted that if the permutation matrix is recovered as $\hat{\Pi}_{\mathrm{ML}}$, then the ML estimation of $\theta$ is as follows:

$$
\hat{\theta}_{\mathrm{ML}}=\frac{h^{T} W^{-1} \hat{\Pi}_{\mathrm{ML}} \bar{y}}{h^{T} W^{-1} h} .
$$

2.1. Clairvoyant Detector from Labeled Observations with the Knowledge of $\theta$. If the parameters $\theta$ and $\Pi$ are both known, the optimal detection statistic in the Neyman-Pearson sense is as follows:

$$
S_{\text {cla }}=\left(\Pi^{T} \tilde{y}\right)^{T} W^{-1} h \theta \underset{\mathscr{H}_{0}}{\stackrel{\mathscr{H}_{1}}{\gtrless}} \gamma,
$$

where the threshold $\gamma$ is chosen such that the false alarm probability $P_{\mathrm{FA}}=\operatorname{Pr}\left(S_{\mathrm{cla}} \geq \gamma \mid \mathscr{H}_{0}\right.$ is true $)$ is set to a desired 
level. This detector is usually referred as the clairvoyant detector, and the corresponding detection probability $P_{D}=$ $\operatorname{Pr}\left(S_{\text {cla }} \geq \gamma \mid \mathscr{H}_{1}\right.$ is true $)$ is as follows:

$$
P_{D}=Q\left(Q^{-1}\left(P_{\mathrm{FA}}\right)-\sqrt{h^{T} W^{-1} h \theta^{2}}\right),
$$

where $Q(x)=\int_{x}^{\infty}(1 / \sqrt{2 \pi}) \exp ^{-t^{2} / 2} d t$ is the tail probability of the standard normal distribution, $Q^{-1}(\cdot)$ denotes its inverse, and $P_{\mathrm{FA}}$ is the false alarm probability. Because the test statistic has the same PDF as the classical meanshifted Gauss-Gauss problem, the deflection coefficient $d^{2}$ characterizes the detection performance and it can be defined as follows:

$$
d^{2}=\frac{\left(E\left(S ; \mathscr{H}_{1}\right)-E\left(S ; \mathscr{H}_{0}\right)\right)^{2}}{\operatorname{var}\left(S ; \mathscr{H}_{0}\right)} .
$$

Here, the deflection coefficient $d^{2}$ of the clairvoyant detector is as follows:

$$
d_{\mathrm{cla}}^{2}=h^{T} W^{-1} h \theta^{2}=\sum_{i=1}^{N} \frac{h_{i}^{2} \theta^{2}}{h_{i}^{2} \sigma_{w}^{2}+\sigma_{v}^{2}}=\theta^{2}\left(\frac{N}{\sigma_{w}^{2}}-\frac{\sigma_{v}^{2}}{\sigma_{w}^{2}} \sum_{i=1}^{N} \frac{1}{h_{i}^{2} \sigma_{w}^{2}+\sigma_{v}^{2}}\right) .
$$

If $N$ is large and $h_{i} \sim N\left(\mu_{h}, \sigma_{h}^{2}\right)$, by Jensen's inequality, we have the following:

$$
\begin{aligned}
h^{T} W^{-1} & =\sum_{i=1}^{N} \frac{h_{i}^{2}}{h_{i}^{2} \sigma_{w}^{2}+\sigma_{v}^{2}}=\frac{N}{\sigma_{w}^{2}}-\frac{\sigma_{v}^{2}}{\sigma_{w}^{2}} \sum_{i=1}^{N} \frac{1}{h_{i}^{2} \sigma_{w}^{2}+\sigma_{v}^{2}} \\
& \approx \frac{N}{\sigma_{w}^{2}}-N \frac{\sigma_{v}^{2}}{\sigma_{w}^{2}} E_{h_{i}}\left[\frac{1}{h_{i}^{2} \sigma_{w}^{2}+\sigma_{v}^{2}}\right] \\
& \leq \frac{N}{\sigma_{w}^{2}}-N \frac{\sigma_{v}^{2}}{\sigma_{w}^{2}} \frac{1}{E_{h_{i}}\left[h_{i}^{2}\right] \sigma_{w}^{2}+\sigma_{v}^{2}}=\frac{N\left(\mu_{h}^{2}+\sigma_{h}^{2}\right)}{\sigma_{v}^{2}+\sigma_{w}^{2}\left(\mu_{h}^{2}+\sigma_{h}^{2}\right)} .
\end{aligned}
$$

Note that the upper bound approximation is tight in the case of $\sigma_{h}^{2}$ being zero.

\subsection{Signal Detection from Labeled Observations without the} Knowledge of $\theta$. For the case of unknown amplitude $\theta$ and known permutation matrix $\Pi$, it can be shown that a uniformly most powerful (UMP) test does not exist. As a consequence, we resort to a suboptimal GLRT detector. The GLRT decides $\mathscr{H}_{1}$ if

$$
\frac{p\left(\Pi^{T} \tilde{y} ; \hat{\theta}, H_{1}\right)}{p\left(\Pi^{T} \tilde{y} ; 0, H_{0}\right)}>\gamma,
$$

where $\hat{\theta}=\left(y^{T} W^{-1} h\right) /\left(h^{T} W^{-1} h\right)$ is the MLE of $\theta$ assuming $\mathscr{H}_{1}$ is true. Consequently, we obtain the GLRT as follows:

$$
S_{\text {glrt }}=\left|\left(\Pi^{T} \tilde{y}\right)^{T} W^{-1} h\right| \underset{\mathscr{H}_{0}}{\stackrel{\mathscr{H}_{1}}{\gtrless}} \gamma,
$$

which is a correlator that accounts for the unknown sign of $\theta$ by taking the sign of its absolute value. The detection probability can be calculated as follows [27]:

$$
\begin{aligned}
P_{D}= & Q\left(Q^{-1}\left(\frac{P_{\mathrm{FA}}}{2}\right)-\sqrt{\theta^{2} h^{T} W^{-1} h}\right) \\
& +Q\left(Q^{-1}\left(\frac{P_{\mathrm{FA}}}{2}\right)+\sqrt{\theta^{2} h^{T} W^{-1} h}\right) .
\end{aligned}
$$

\section{Detection with Permuted Observations}

In this section, we investigate the detection problem (7) under the circumstance that both the desired parameter $\theta$ and nuisance parameter $\Pi$ are unknown. In order to detect the presence of the signal, the recovery of the permutation matrix is indispensable. But the joint optimization of both unknowns is difficult due to the nonconvexity of $\Pi$. To circumvent the dilemma, the joint optimization problem will be decomposed into two subproblems. To be more specific, previously, we first analyze the problem of detection with labeled observations in Section 2.1 and Section 2.2. Now in Subsection 3.1, we solve the detection problem from permuted observations with the knowledge of $\theta$. Finally in Subsection 3.2, the complete detection problem (7) is studied.

3.1. Signal Detection from Permuted Observations with Knowledge of $\theta$. Now we consider the case that the signal $\theta$ and the channel coefficients are known while the observations collected from the sensors are permutated. Under this assumption, we first propose an approximated GLRT as follows [27]:

$$
\begin{aligned}
S_{w, \mathrm{glrt}}= & \min _{\Pi \in P_{n}}\left(\Pi^{T} \tilde{y}\right)^{T} W^{-1}\left(\Pi^{T} \tilde{y}\right) \\
& -\min _{\Pi \in P_{n}}\left(\Pi^{T} \tilde{y}-h \theta\right)^{T} W^{-1}\left(\Pi^{T} \tilde{y}-h \theta\right) \underset{\mathscr{C}_{0}}{\stackrel{\mathscr{H}_{1}}{\gtrless}} \gamma,
\end{aligned}
$$

where the searching space of possible permutation matrix set is $N$ !. Under either hypothesis $\mathscr{H}_{0}$ or $\mathscr{H}_{1},\left(\Pi^{T} \tilde{y}\right)^{T} W^{-1}\left(\Pi^{T} \tilde{y}\right)$ or

$$
\left(\Pi^{T} \tilde{y}-h \theta\right)^{T} W^{-1}\left(\Pi^{T} \tilde{y}-h \theta\right) \underset{\mathscr{H}_{0}}{\stackrel{\mathscr{H}_{1}}{\gtrless}} \gamma
$$

can be decomposed as $\sum_{i=0}^{N-1} f\left(\theta, y_{i}, h_{i}\right)$. For problem $\min _{\Pi \in P_{n}}\left(\Pi^{T} \tilde{y}\right)^{T} W^{-1}\left(\Pi^{T} \tilde{y}\right)$, it can be easily shown that the permutation matrix can be found by sorting $\tilde{y}^{2}$ (element-wise square) according to $\mathbf{h}^{2}$. For problem

$$
\min _{\Pi \in P_{n}}\left(\Pi^{T} \tilde{y}-h \theta\right)^{T} W^{-1}\left(\Pi^{T} \tilde{y}-h \theta\right) \underset{\mathscr{H}_{0}}{\stackrel{\mathscr{H}_{1}}{\gtrless}} \gamma
$$

which is decomposed as $\sum_{i=0}^{N-1} f\left(\theta, y_{i}, h_{i}\right)$, one can swap the elements of $\tilde{y}$ to minimize the objective function for a given $\theta$ to obtain a smaller objective function value, which involves $O\left(N^{2}\right)$ computation complexity. 
In order to construct a more computational efficient detector, the linear approach is further incorporated to eliminate the effects of the permutation matrix. Observe that given any permutation matrix $\Pi_{1}, \Pi_{2}$ and observation $\tilde{y}$, $\mathbf{A} \Pi_{1}=\mathbf{A} \Pi_{2}$ holds if the rows of the matrix $\mathbf{A}$ have constant entries. As a consequence, the linear approach reduces to the simple mean detector as follows:

$$
S_{\text {mean }}=\frac{1}{N} \sum_{i=1}^{N}[\tilde{y}]_{i} \underset{\mathscr{H}_{0}}{\stackrel{\mathscr{H}_{1}}{\gtrless}} \gamma,
$$

where

$$
\begin{aligned}
& H_{0}: S_{\text {mean }} \sim N\left(0, \frac{\operatorname{tr}(W)}{N^{2}}\right), \\
& H_{1}: S_{\text {mean }} \sim N\left(\frac{1}{N} \sum_{i=0}^{N-1} h_{i} \theta, \frac{\operatorname{tr}(W)}{N^{2}}\right) .
\end{aligned}
$$

The false alarm and detection probability are, respectively, as follows:

$$
\begin{aligned}
P_{\mathrm{FA}} & =\operatorname{Pr}\left(S_{\text {mean }} \geq \gamma \mid \mathscr{H}_{0} \text { is true }\right)=Q\left(\frac{\gamma}{\sqrt{\operatorname{tr}(W) / N^{2}}}\right) \\
& =Q\left(\frac{\gamma}{\sqrt{\left(1 / N^{2}\right) \sum_{i=0}^{N-1} h_{i}^{2} \sigma_{w}^{2}+\left(\sigma_{v}^{2} / N\right)}}\right),
\end{aligned}
$$

$$
\begin{aligned}
P_{D} & =\operatorname{Pr}\left(S_{\text {mean }} \geq \gamma \mid \mathscr{H}_{1} \text { is true }\right)=Q\left(\frac{\gamma-\left|(1 / N) \sum_{i=0}^{N-1} h_{i} \theta\right|}{\sqrt{\operatorname{tr}(W) / N^{2}}}\right) \\
& =Q\left(\frac{\gamma-(1 / N)\left|\sum_{i=0}^{N-1} h_{i} \theta\right|}{\sqrt{\left(1 / N^{2}\right) \sum_{i=0}^{N-1} h_{i}^{2} \sigma_{w}^{2}+\left(\sigma_{v}^{2} / N\right)}}\right) .
\end{aligned}
$$

Moreover, the receiver operating characteristic (ROC) of the detector can be expressed as follows:

$$
P_{D}=Q\left(Q^{-1}\left(P_{\mathrm{FA}}\right)-\frac{\left|\sum_{i=0}^{N-1} h_{i} \theta\right|}{\sum_{i=0}^{N-1} h_{i}^{2} \sigma_{w}^{2}+N \sigma_{v}^{2}}\right) .
$$

Define

$$
\begin{aligned}
d_{\text {mean }}^{2} & =\frac{\left|\sum_{i=0}^{N-1} h_{i} \theta\right|}{\sqrt{\sum_{i=0}^{N-1} h_{i}^{2} \sigma_{w}^{2}+N \sigma_{v}^{2}}} \approx \frac{N E_{h_{i}}\left[h_{i}\right] \theta}{\sqrt{N E_{h_{i}}\left[h_{i}^{2}\right] \sigma_{w}^{2}+N \sigma_{v}^{2}}} \\
& =\sqrt{\frac{N \mu_{h}^{2} \theta^{2}}{\left(\mu_{h}^{2}+\sigma_{h}^{2}\right) \sigma_{w}^{2}+\sigma_{v}^{2}}} .
\end{aligned}
$$

From (13) and (25), the SNR loss incurred by the mean detector w.r.t. the clairvoyant is obtained as follows:

$$
\mathscr{L}_{\text {mean } / \text { cla }}=1-\left(\frac{d_{\text {mean }}^{2}}{d_{\text {cla }}^{2}}\right) \approx \frac{\sigma_{h}^{2}}{\mu_{h}^{2}+\sigma_{h}^{2}} .
$$

If $\sigma_{h}^{2}=0$, i.e., $\mathbf{h}$ is a constant vector, the loss $\mathscr{L}_{\text {mean/cla }}$ is zero, which implies that the detector uses the mean statistic performer as well as the optimal clairvoyant detector. This is obvious because the permutation has no effect on the observation given a constant $\mathbf{h}$. For zero mean vector $\mathbf{h}\left(\mu_{h}=0\right)$, the loss achieves its maximum 1 . This is because the mean detector can not detect the signal given the mean of $\mathbf{h}$ is zero. Before ending the present subsection, we slightly digress to emphasize that the result presented in (26) is accurate for $\sigma_{w}^{2}=0$, while holds approximately for the general nonzero small $\sigma_{w}^{2}$.

\subsection{Signal Detection from Permuted Observations without} Knowledge of $\theta$. For the complete detection problem (7), we should jointly optimize the parameters $\theta$ and $\Pi$. In [20], an alternating minimization algorithm is proposed to find the maximum likelihood estimation of $\theta$ and $\Pi$. The alternating minimization algorithm works as follows: Given $\hat{\theta}^{t-1}$, we update the permutation matrix and obtain $\hat{\Pi}^{t-1}$. Then we fix the permutation matrix as $\hat{\Pi}^{t-1}$ and update $\theta$. The alternative steps are repeated until the algorithm converges. In this paper, we use the above approach to evaluate the performance of an approximation of the GLRT as follows:

$$
\begin{aligned}
S_{\mathrm{wo}, \mathrm{glrt}}= & \min _{\Pi \in P_{n}}\left(\Pi^{T} \tilde{y}\right)^{T} W^{-1}\left(\Pi^{T} \tilde{y}\right) \\
& -\min _{\Pi \in P_{n}, \theta}\left(\Pi^{T} \tilde{y}-h \theta\right)^{T} W^{-1}\left(\Pi^{T} \tilde{y}-h \theta\right) \underset{\mathscr{H}_{0}}{\stackrel{\mathscr{H}_{1}}{\gtrless}} \gamma .
\end{aligned}
$$

Notice that although the optimality of the proposed algorithm is not guaranteed, the algorithm appears to work well when the desired parameter $\theta$ is accurately estimated. As the number of sensors $N$ increases, the probability of perfect permutation matrix recovery decreases [18]. On the other hand, numerical simulations have shown that the accuracy of the estimation of $\theta$ improves with increasing $N$ [20]. As a consequence, it is interesting to evaluate the performance in terms of $N$, which will be addressed by performing numerical experiments in the following section.

In [28], it has been proved that in the absence of additive noise $\mathbf{V}$, the permutation matrix can be recovered exactly. Taking into consideration that

$$
\begin{aligned}
\tilde{y}^{T} \tilde{y} & =\left(\Pi^{T} \tilde{y}\right)^{T} \Pi^{T} \tilde{y}=(h \theta+e)^{T}(h \theta+e)=\theta^{2} h^{T} h+e^{T} e+2 \theta h^{T} e \\
& \approx\left(\frac{\theta^{2} h^{T} h+h^{T} h \sigma_{w}^{2}}{K}\right)+\left(\frac{N \sigma_{v}^{2}}{K}\right),
\end{aligned}
$$


a reasonable initial point of $\theta$ can be chosen as follows:

$$
\theta_{\text {init }}= \pm \frac{\sqrt{\max \left(\|\tilde{y}\|^{2}-\left(N \sigma_{v}^{2}+\sigma_{w}^{2}\|h\|^{2}\right) / K, 0\right)}}{\|h\|} .
$$

We then use the initial estimation $\theta_{\text {init }}$ to iteratively update the permutation matrix and unknown $\theta$.

In addition, if the channel information is also missing, the test statistic would be a function of observations $y$ such as the previous mean detector. Utilizing the second order information yields the following energy detector:

$$
S_{\text {ene }}=\|\tilde{y}\|_{2}^{2} \underset{\mathscr{H}_{0}}{\stackrel{\mathscr{H}_{1}}{\gtrless}} \gamma .
$$

The performance of the energy detector will also be evaluated via Monte Carlo (MC) simulations.

\section{Numerical Simulation}

In this section, various numerical simulations are conducted to evaluate the performances of the proposed detectors; the first three experiments are performed in both the high noise level and low noise level scenarios, separately. For the first simulation, the results are presented in the form of ROC curves, i.e., $P_{\mathrm{D}}$ versus $P_{\mathrm{FA}}$. Next, the relationship between the detection probability $P_{\mathrm{D}}$ and the observation time $K$ is investigated, where the false alarm probability is set to a desired level. Then, the detection probability $P_{\mathrm{D}}$ versus the number of the sensors $N$ is studied, where the false alarm probability is also set to a desired level. Finally, the probability $P_{\text {Per }}$ of successfully recovering the permutation matrix $\Pi$ versus the noise level $\mathrm{SNR}_{\mathrm{w}}$ and $\mathrm{SNR}_{v}$ is demonstrated, where $\mathrm{SNR}_{w}=10 \log _{10}\left(\theta^{2} / \sigma_{w}^{2}\right), \mathrm{SNR}_{v}=10 \log _{10}\left(\theta^{2} / \sigma_{v}^{2}\right)$, and $P_{\mathrm{Per}}$ is defined as follows:

$$
P_{\text {Per }}=\operatorname{Pr}\left(\hat{\Pi} \neq \Pi_{0} \mid \mathscr{H}_{1} \text { is true }\right),
$$

where $\hat{\Pi}$ is the estimated permutation matrix, and $\Pi_{0}$ denotes the true one. We set $\theta=1$ unless stated otherwise. The channel coefficients $h_{i}$ are drawn from the i.i.d. Gaussian distribution $N\left(\mu_{h}, \sigma_{h}^{2}\right)$ and $\left\{h_{i}\right\}_{i=1}^{N}$ is averaged such that $1 / N \sum_{i=1}^{N} h_{i}=\mu_{h}$. The performances of various detectors except the clairvoyant and the labeled GLRT detectors are evaluated via $\mathrm{MC}$ simulations, and the trial number of $\mathrm{MC}$ simulations is 10,000 . For simplicity, the approximations of the detectors are referred without the prefix "approximation" in this section.

\subsection{ROC Curves}

4.1.1. High Noise Level. The results are presented in Figure 1 with various values of $\mu_{h}$ and $\sigma_{h}^{2}$ under high noise level scenario. The parameters are set as follows: $N=20, K=5$, $\sigma_{w}^{2}=10$, and $\sigma_{v}^{2}=10$. The four subgraphs in Figure 1 refer to $\left(\mu_{h}, \sigma_{h}^{2}\right)=(1,1),\left(\mu_{h}, \sigma_{h}^{2}\right)=(0,1),\left(\mu_{h}, \sigma_{h}^{2}\right)=(1,0)$, and $\left(\mu_{h}, \sigma_{h}^{2}\right)=(1,10)$, respectively. From Figure $1(\mathrm{a})$, it can be seen that the labeled GLRT detector performs similarly to that of the unlabeled detector with the knowledge of $\theta$; both detectors detect better than unlabeled detector without the knowledge of $\theta$, which demonstrates that the label information play an important role in reliable signal detection when the variance is comparable to the mean of $\mathbf{h}$. For unlabeled detectors, it can be shown that the mean detector is very attractive because of its simple implementation and competitive performance. It is also noted that the unlabeled detector with known $\theta$ has a similar detection performance to that of the mean detector. From Figure 1(b), it can be seen that the performance of the energy detector is comparable to the other unlabeled data-based detectors; all these three detectors perform inferior to that of the labeled detector. Another observation gleaned from Figure 1(a) is that the mean detector is the worst detector for zero mean channel coefficients, which coincides well with the theoretical analysis presented in Section 2. In the case of constant channel coefficients, the clairvoyant detector, the mean detector, and the unlabeled GLRT with known $\theta$ detector have equal performance, and the performance of the labeled GLRT detector exhibits the same performance as the unlabeled GLRT with unknown $\theta$ detector, as can be seen from Figure 1(c). What we can conclude from Figure 1(d) is that the unlabeled GLRT detector without the knowledge of $\theta$ has the similar performance to that of the mean detector, and both detectors perform worse than the unlabeled detector with the known $\theta$.

4.1.2. Low Noise Level. The simulation parameters are the same as that of the above high noise level except $\sigma_{w}^{2}=4$, $\sigma_{v}^{2}=4$. When the noise level is low, the conclusions from Figure 2 are basically the same as those of the high noise level. But the detection probability $P_{\mathrm{D}}$ reaches 1 more quickly with the benefit of the improved SNR.

\section{2. $P_{D}$ versus $K$}

4.2.1. High Noise Level. Next, we investigate the detection performances versus the observation time $K$ in the scenario of high noise level, where $N=40, P_{\mathrm{FA}}=0.05, \sigma_{w}^{2}=10$, and $\sigma_{v}^{2}=10$. Since increasing $K$ will decrease the variance of the equivalent noise e defined in (7), it can be expected that the detection performance of almost all the detectors will improve as $K$ increases. Actually, numerical experiments indicate the intuitive conjecture (see Figure 3). The only exception is that the mean detector does not work under zero-mean channel coefficients; therefore, increasing $K$ does not bring any gains. It is also noted that the detection probabilities of the mean detector and the energy detector occasionally decrease in subgraph (d) with the increase of $K$.

4.2.2. Low Noise Level. The simulation parameters are the same as those of the high noise level except $\sigma_{w}^{2}=6, \sigma_{v}^{2}=6$. When the noise level is low, the conclusions from Figure 4 are basically the same as those of the high noise level. But the detection probability $P_{\mathrm{D}}$ reaches 1 more quickly and the curves are more smooth and monotonous. 


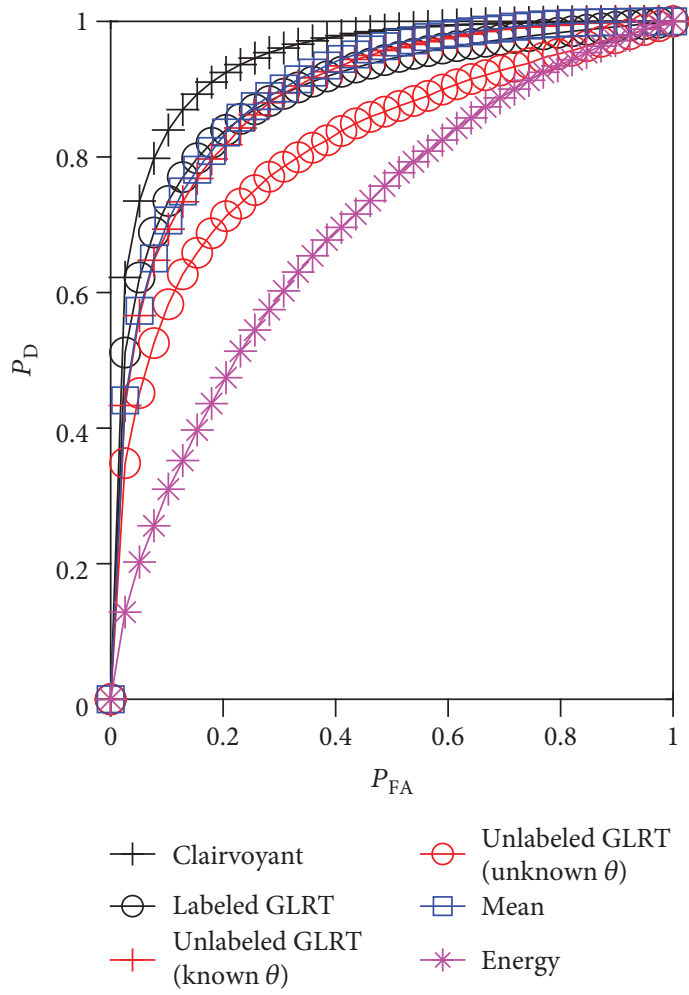

(a)

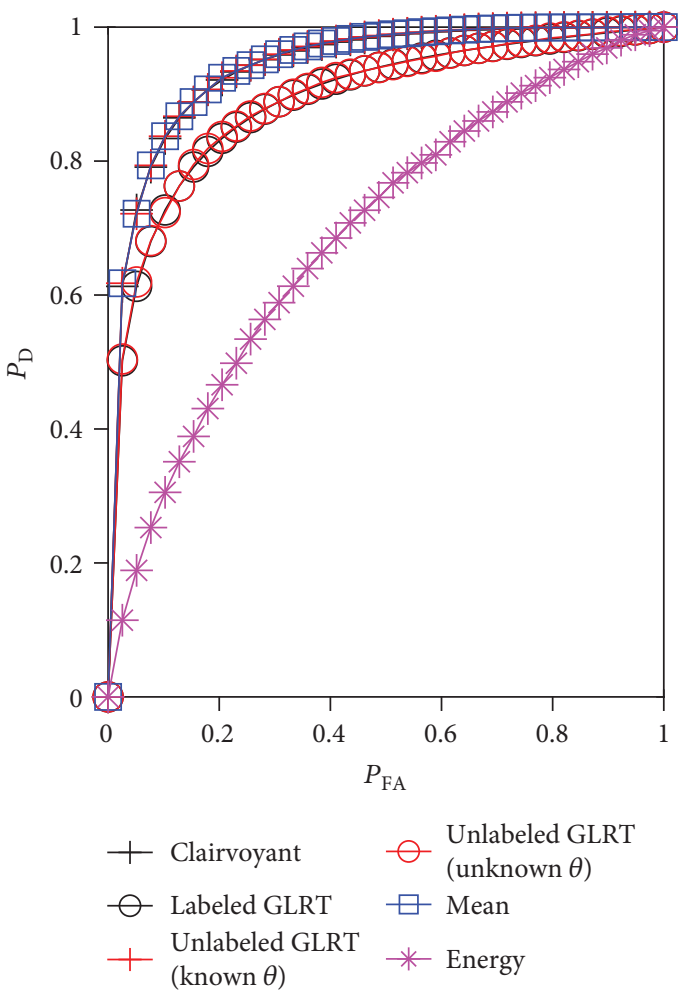

(c)

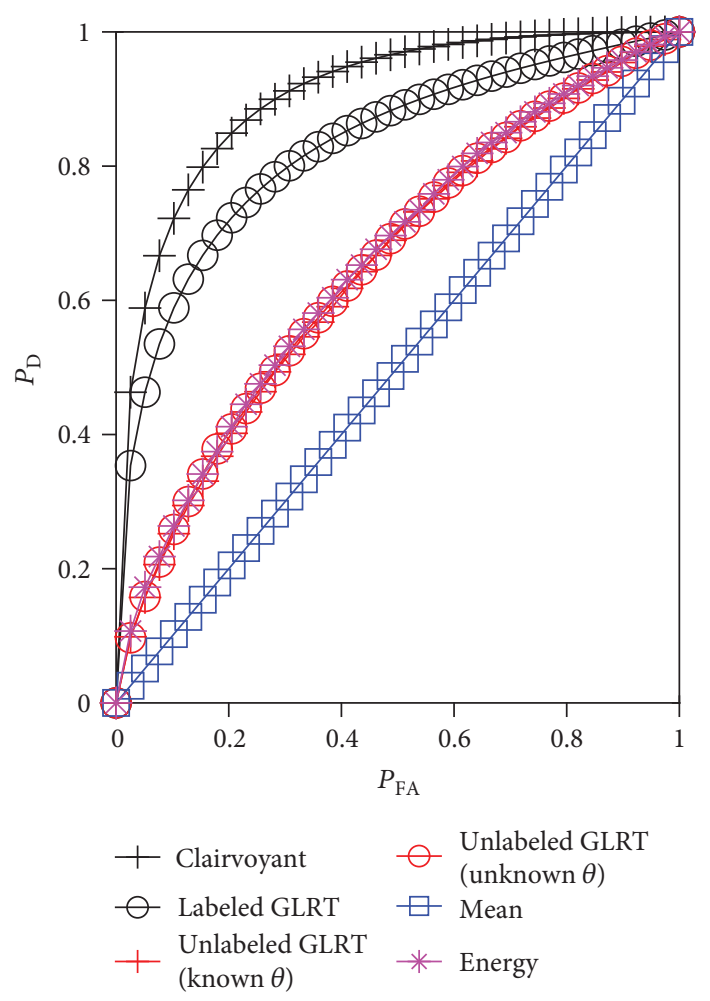

(b)

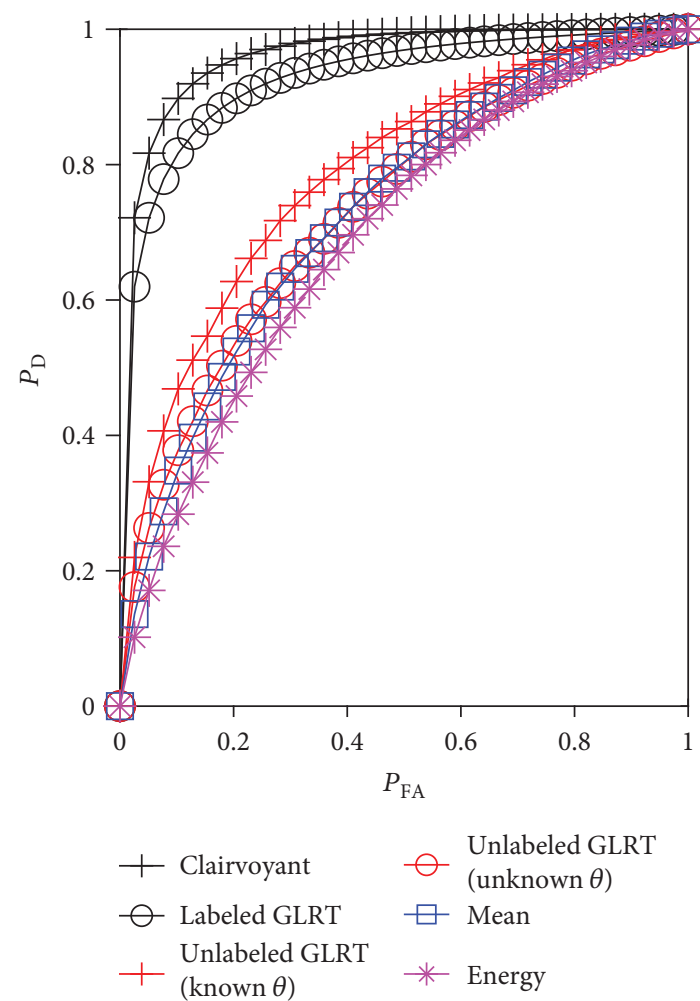

(d)

Figure 1: ROC of various detectors with $\left(\mu_{h}, \sigma_{h}^{2}\right)=(1,1)(\mathrm{a}),(0,1)(\mathrm{b}),(1,0)(\mathrm{c})$, and $(1,10)(\mathrm{d})$ with $\sigma_{w}^{2}=10, \sigma_{v}^{2}=10$. 


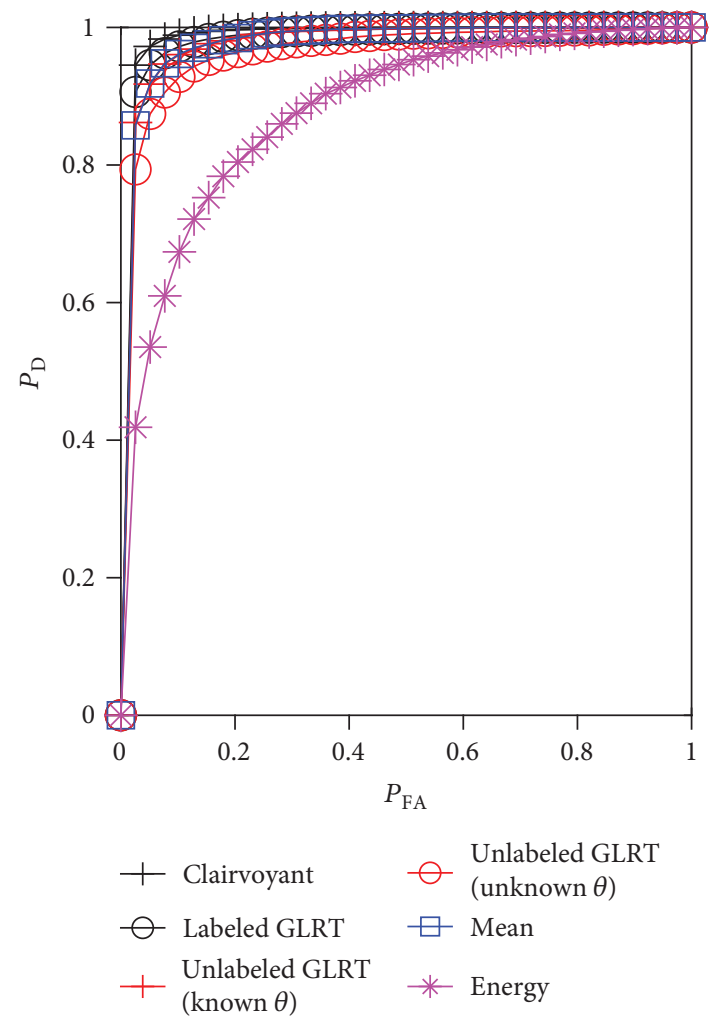

(a)

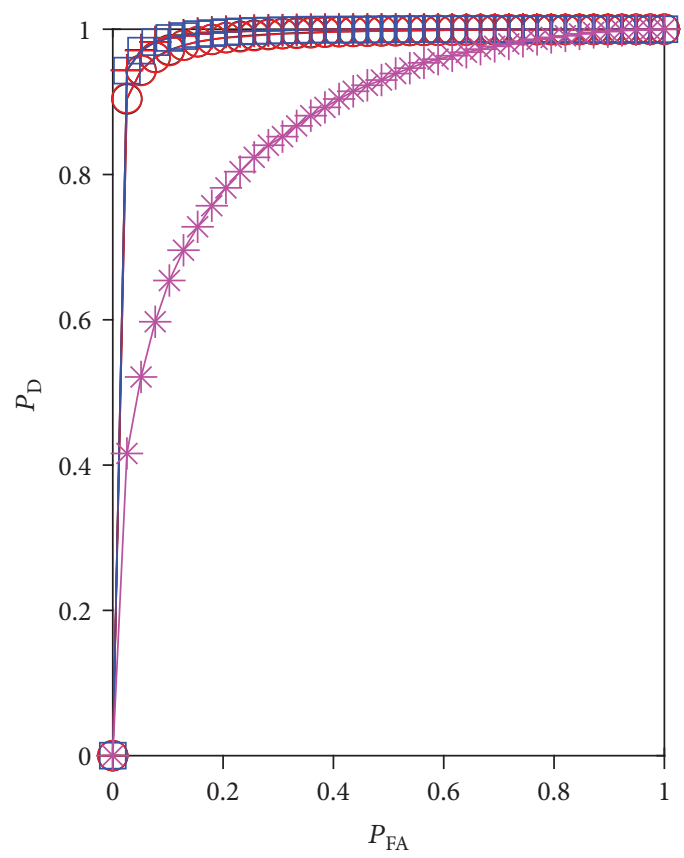

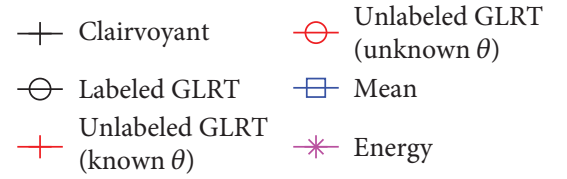

(c)

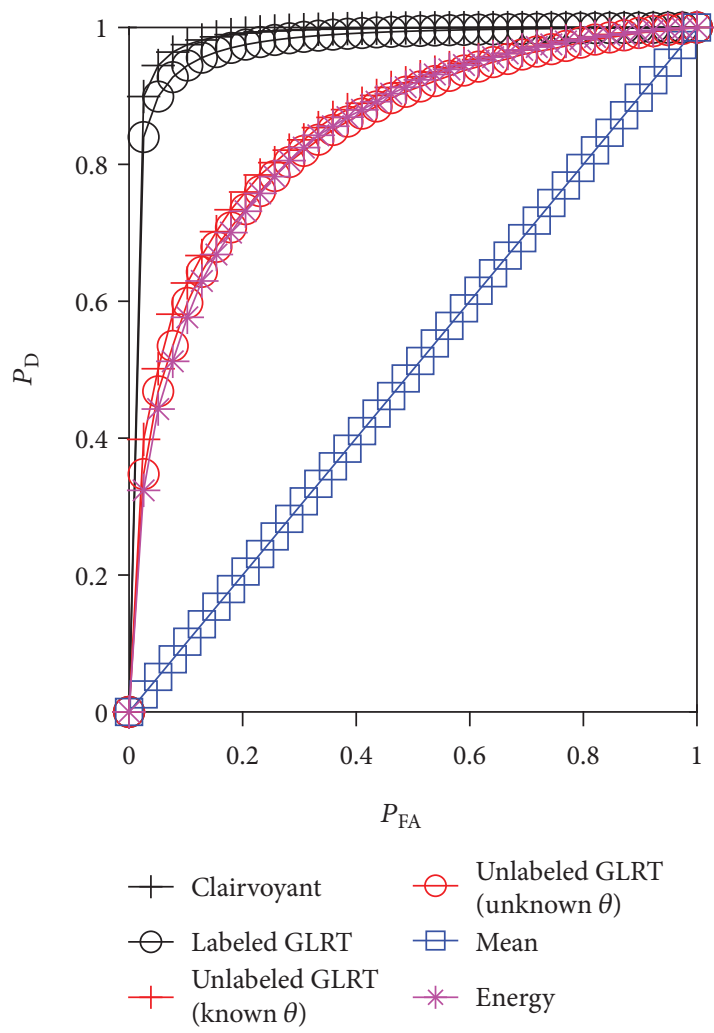

(b)

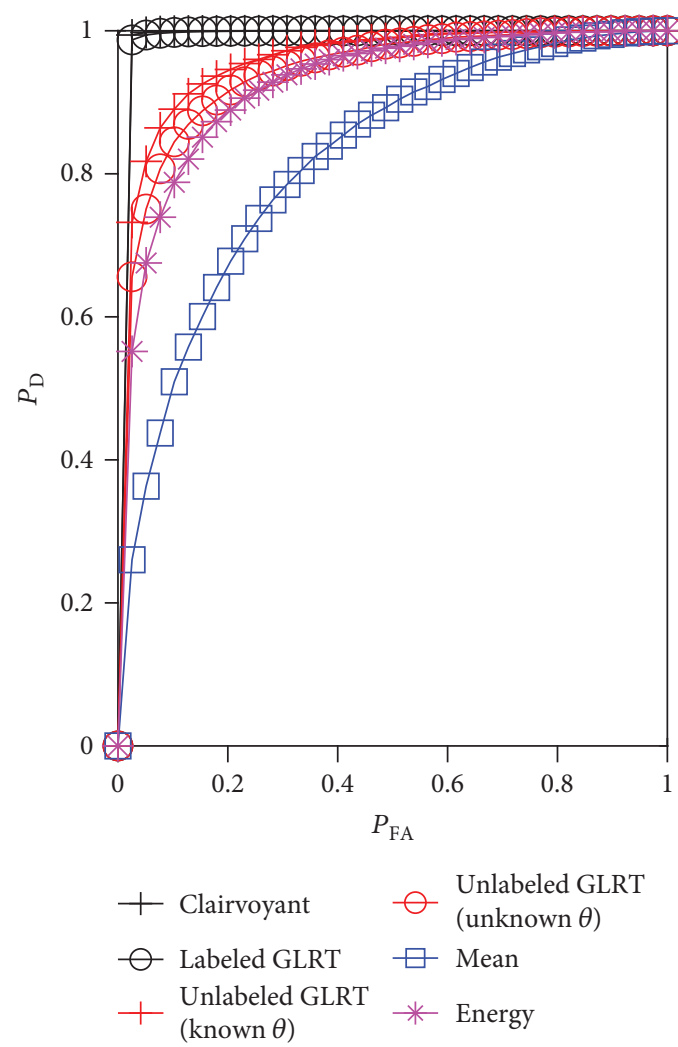

(d)

FigURE 2: ROC of various detectors with $\left(\mu_{h}, \sigma_{h}^{2}\right)=(1,1)(\mathrm{a}),(0,1)(\mathrm{b}),(1,0)(\mathrm{c})$, and $(1,10)(\mathrm{d})$ with $\sigma_{w}^{2}=4, \sigma_{v}^{2}=4$. 


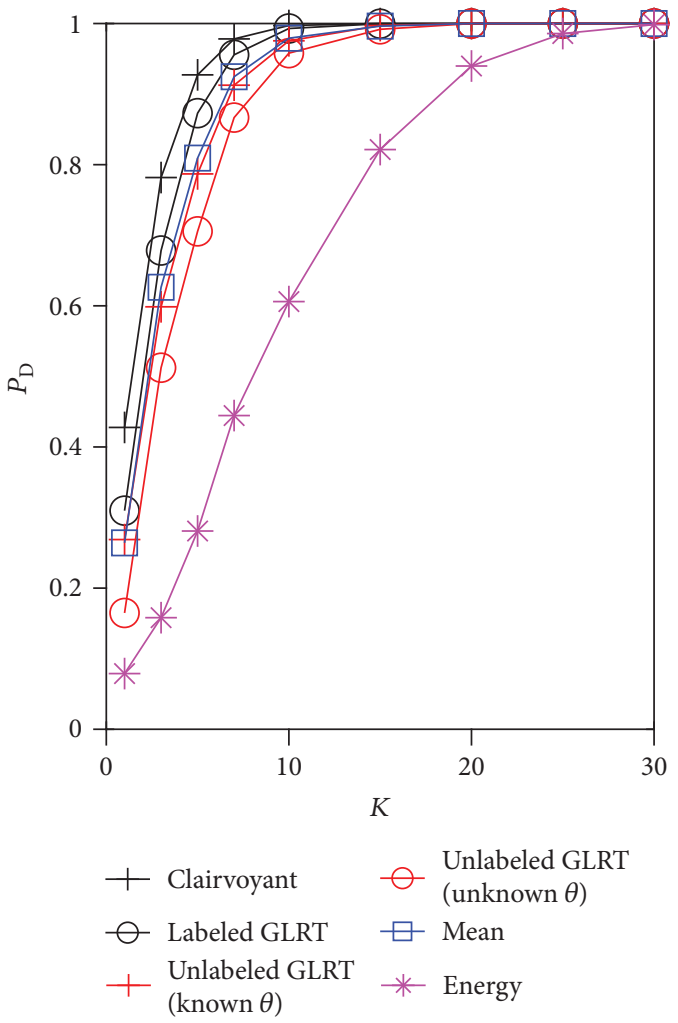

(a)

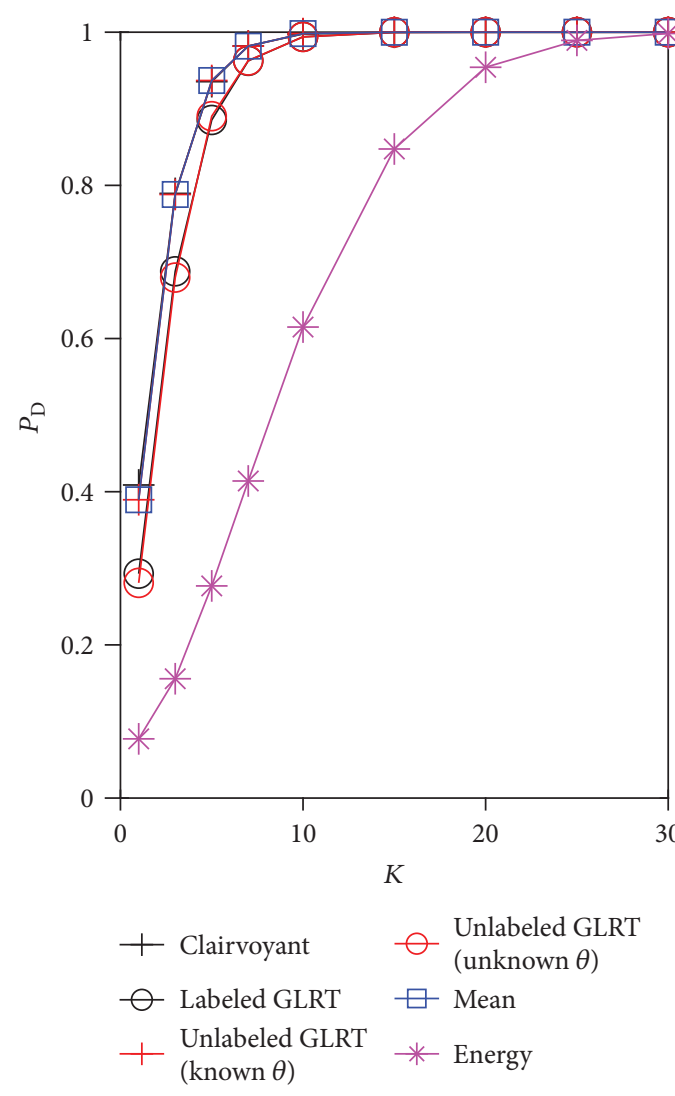

(c)
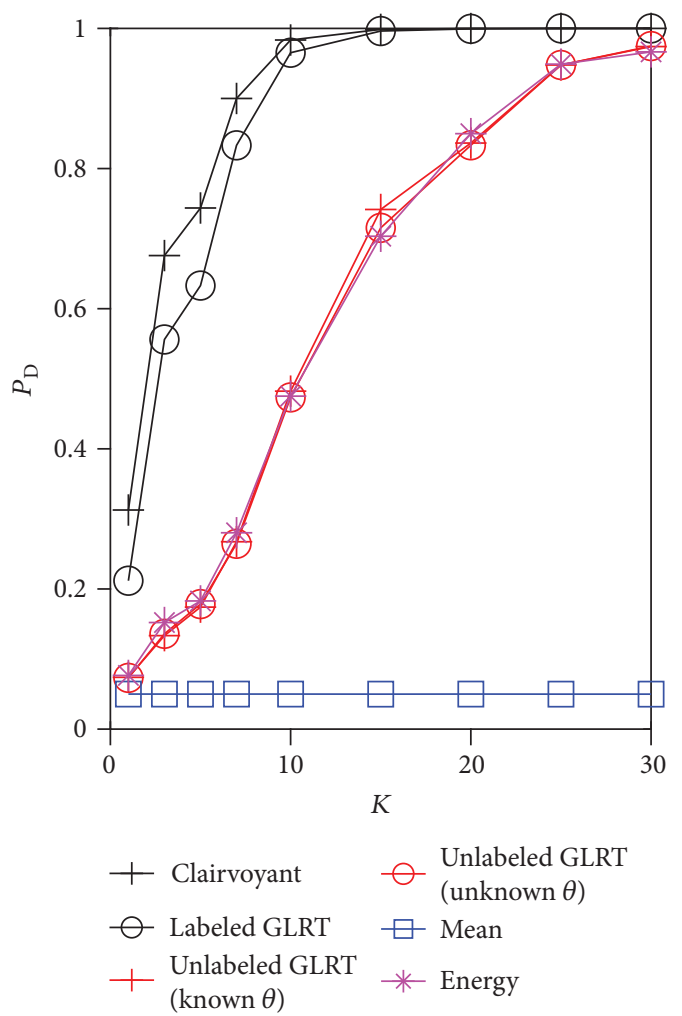

(b)

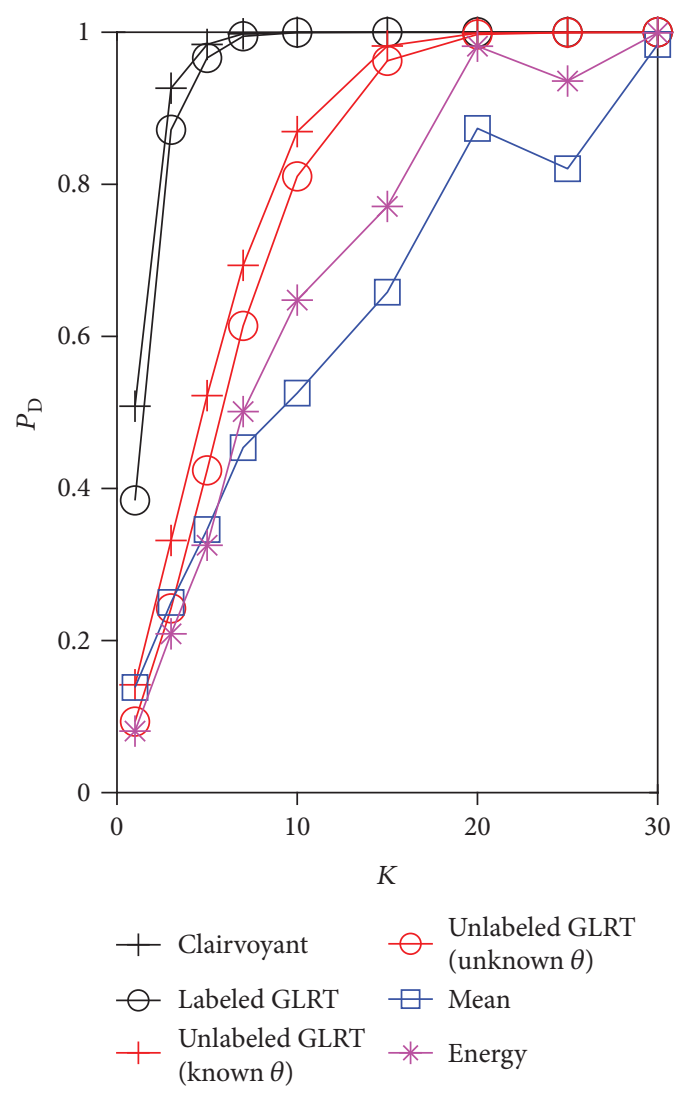

(d)

Figure 3: $P_{\mathrm{D}}$ of different detectors versus $K$ with $\left(\mu_{h}, \sigma_{h}^{2}\right)=(1,1)(\mathrm{a}),(0,1)(\mathrm{b}),(1,0)(\mathrm{c})$, and $(1,10)(\mathrm{d})$ with $\sigma_{w}^{2}=10, \sigma_{v}^{2}=10$. 


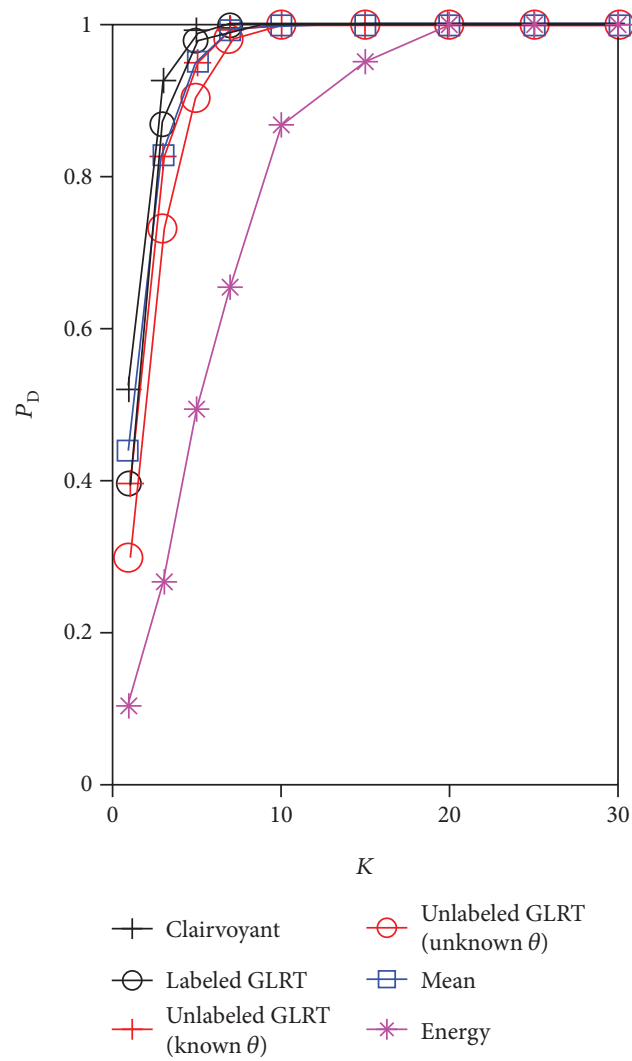

(a)

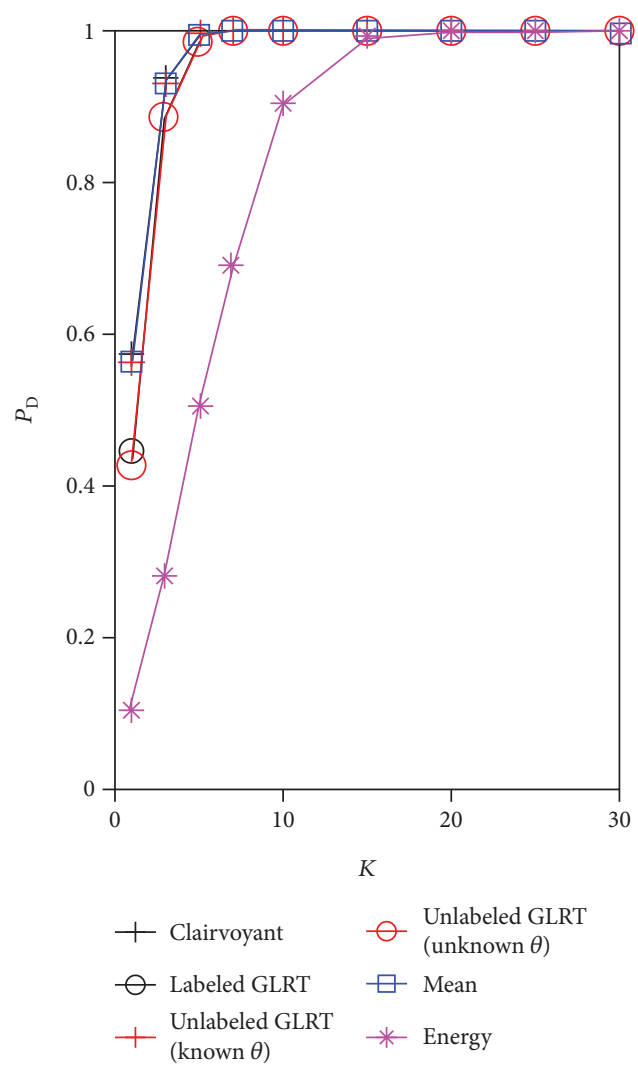

(c)

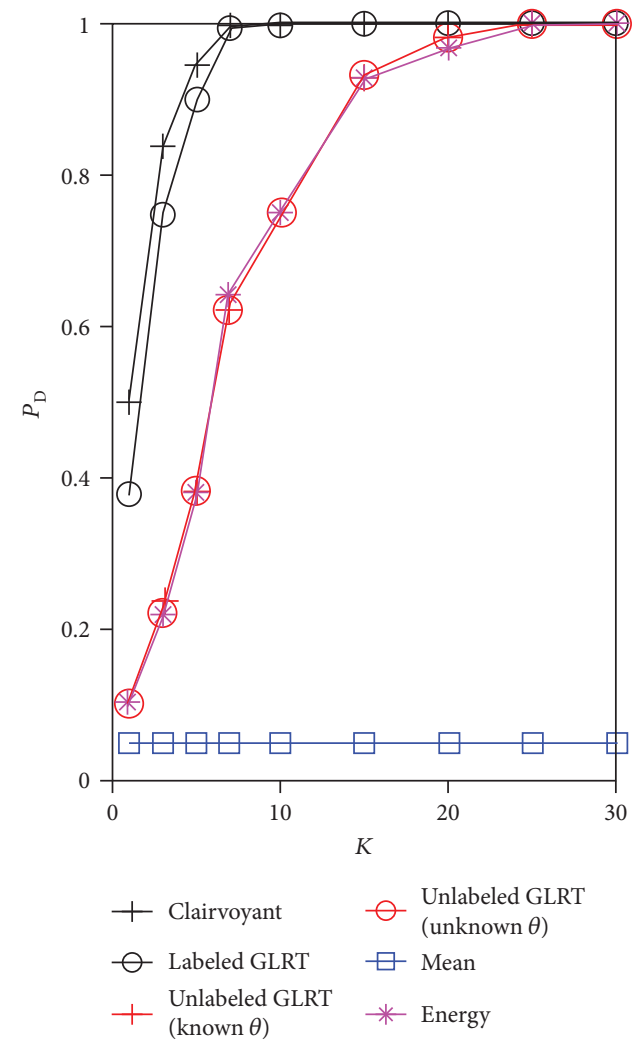

(b)

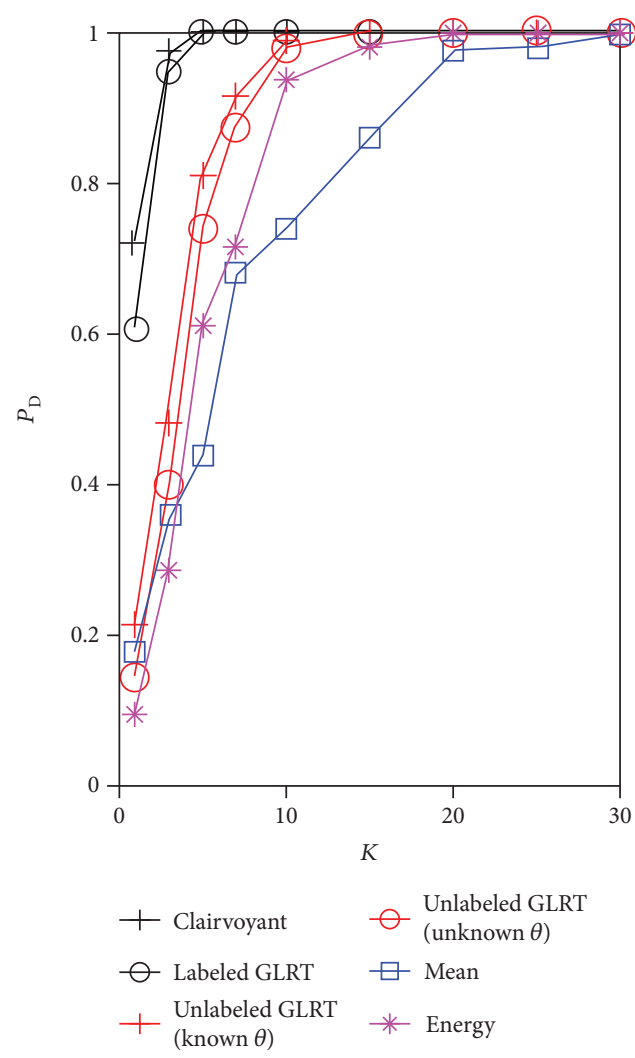

(d)

FIGURE 4: $P_{\mathrm{D}}$ of different detectors versus $K$ with $\left(\mu_{h}, \sigma_{h}^{2}\right)=(1,1)(\mathrm{a}),(0,1)(\mathrm{b}),(1,0)(\mathrm{c})$, and $(1,10)(\mathrm{d})$ with $\sigma_{w}^{2}=6, \sigma_{v}^{2}=6$. 

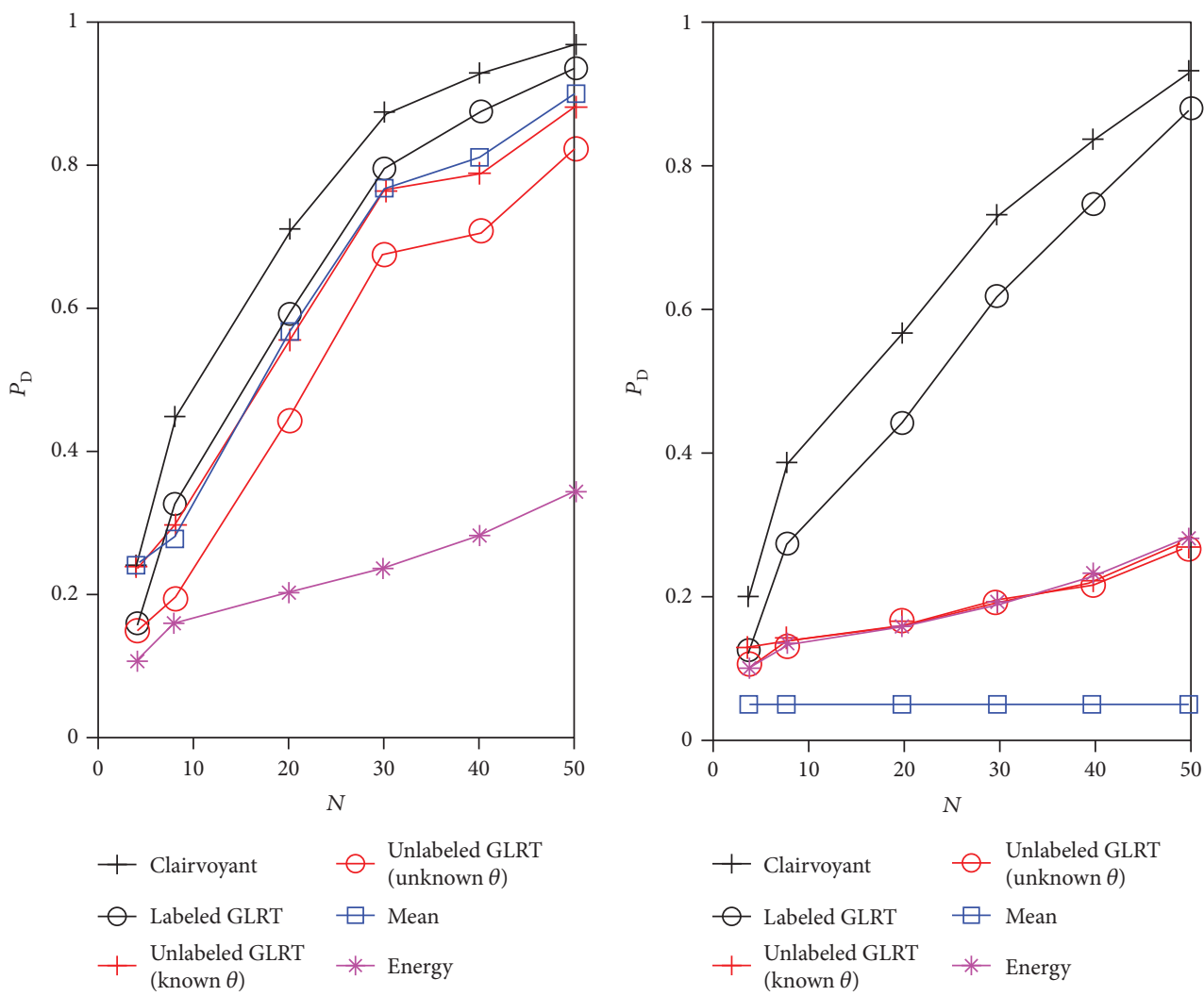

(a)

(b)
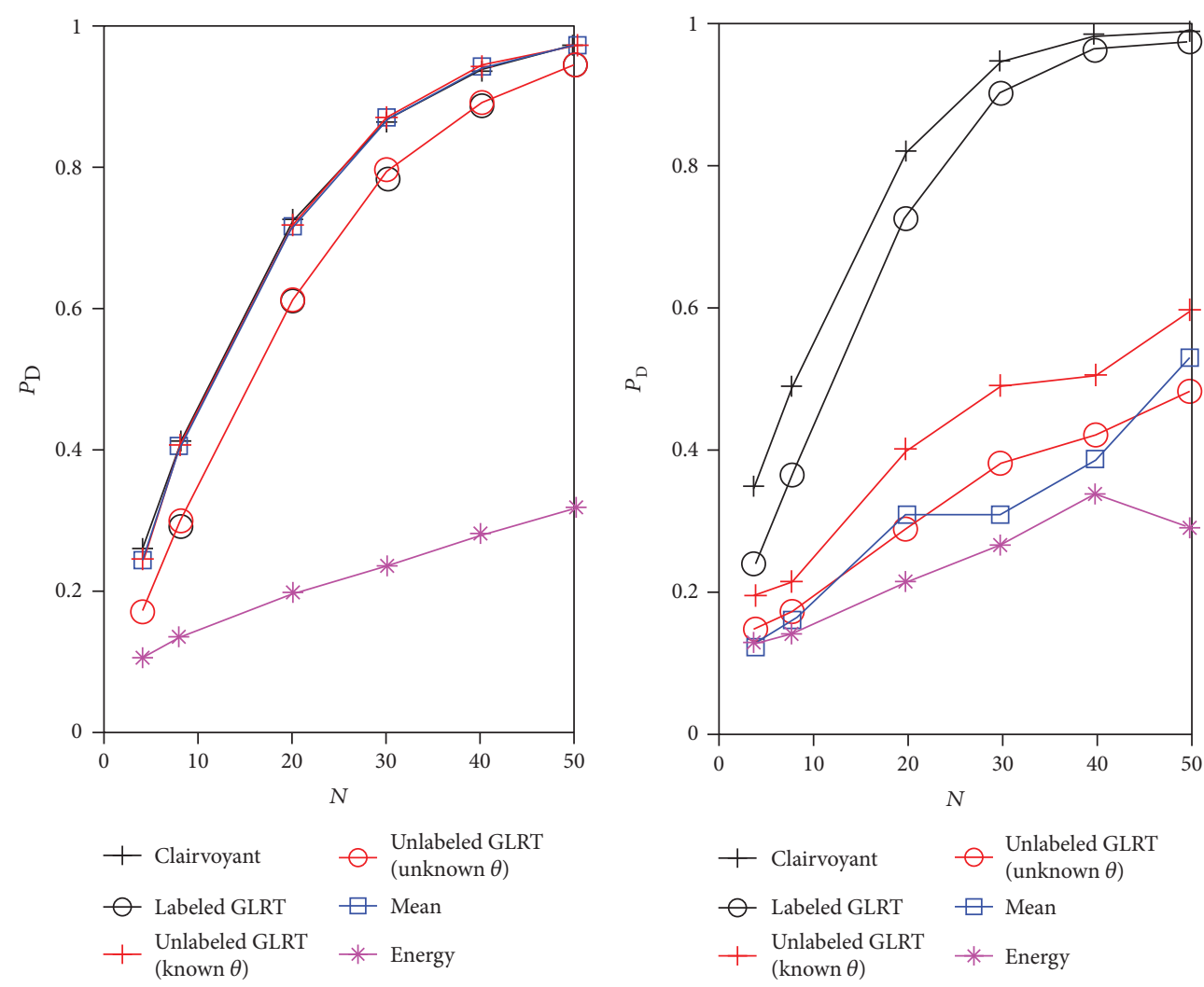

(c)

(d)

Figure 5: $P_{\mathrm{D}}$ of different detectors versus $N$ with $\left(\mu_{h}, \sigma_{h}^{2}\right)=(1,1)(\mathrm{a}),(0,1)(\mathrm{b}),(1,0)(\mathrm{c})$, and $(1,10)(\mathrm{d})$ with $\sigma_{w}^{2}=10, \sigma_{v}^{2}=10$. 


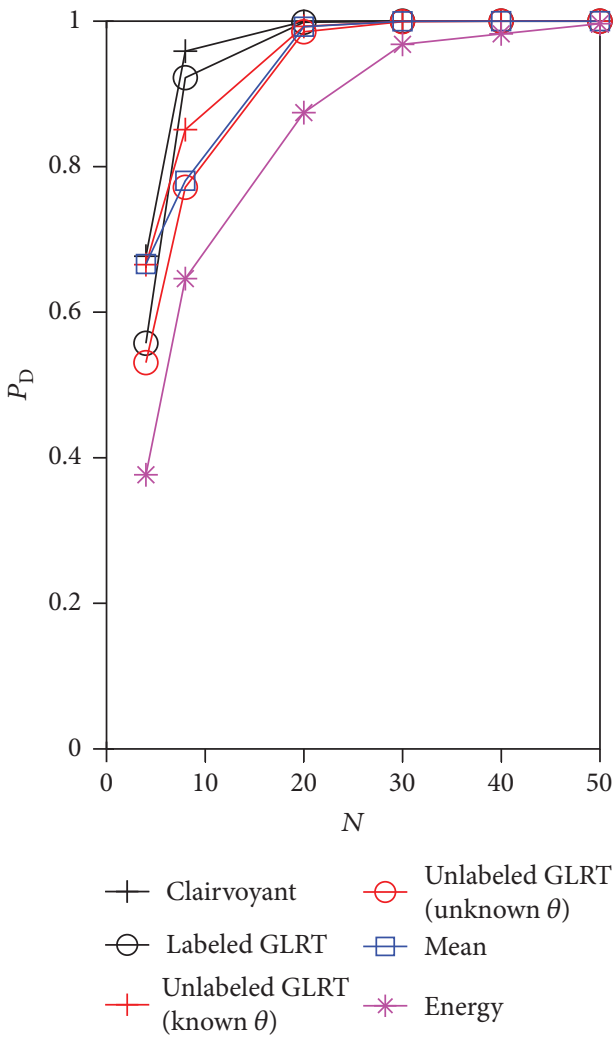

(a)

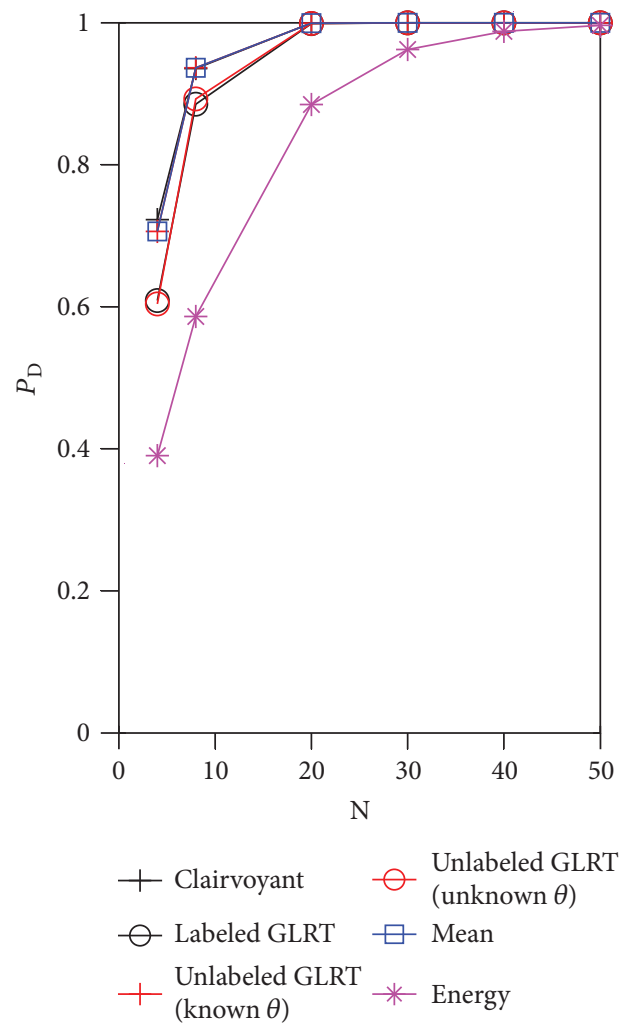

(c)

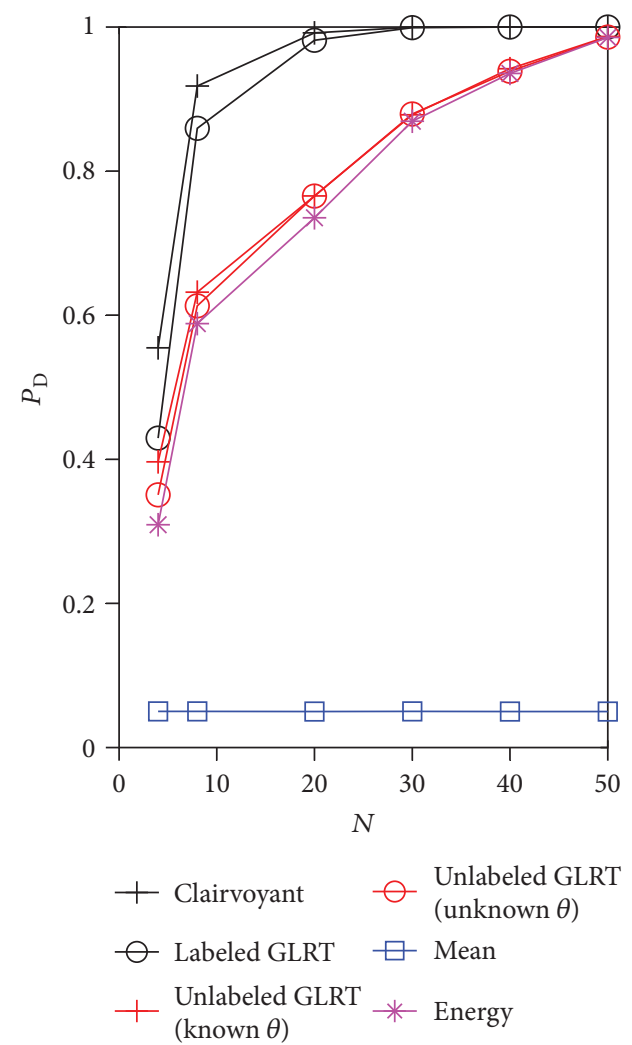

(b)

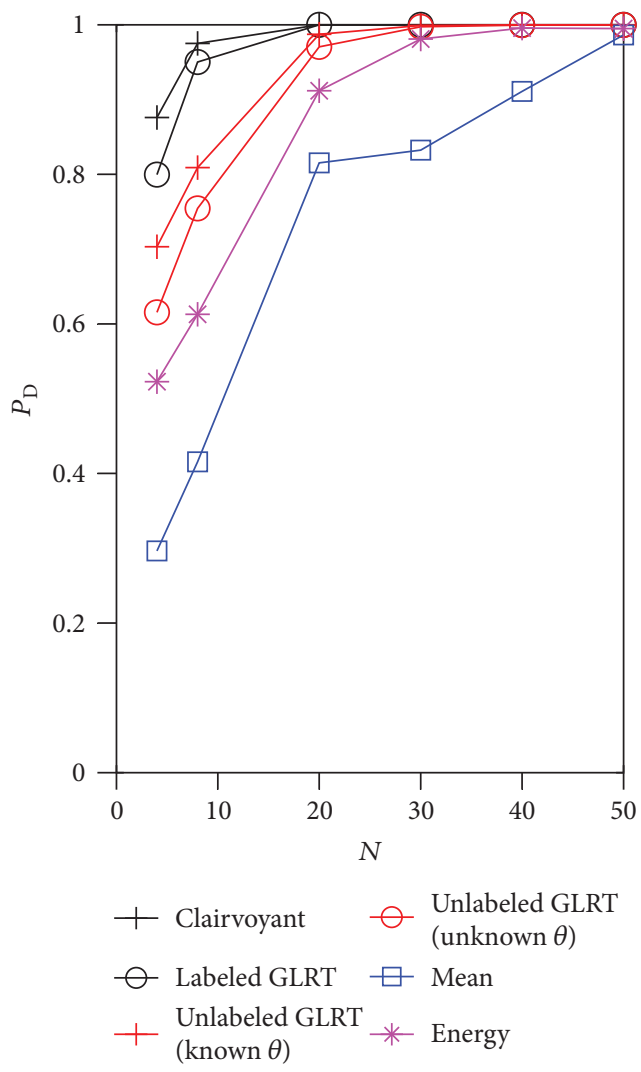

(d)

Figure 6: $P_{\mathrm{D}}$ of different detectors versus $N$ with $\left(\mu_{h}, \sigma_{h}^{2}\right)=(1,1)(\mathrm{a}),(0,1)(\mathrm{b}),(1,0)(\mathrm{c})$, and $(1,10)(\mathrm{d})$ with $\sigma_{w}^{2}=2, \sigma_{v}^{2}=2$. 


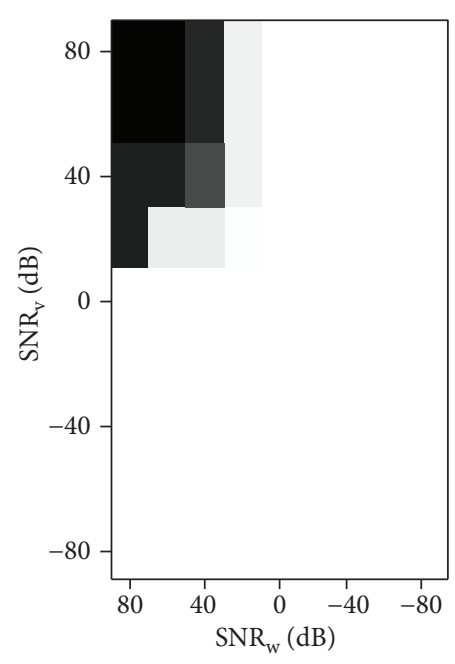

(a)
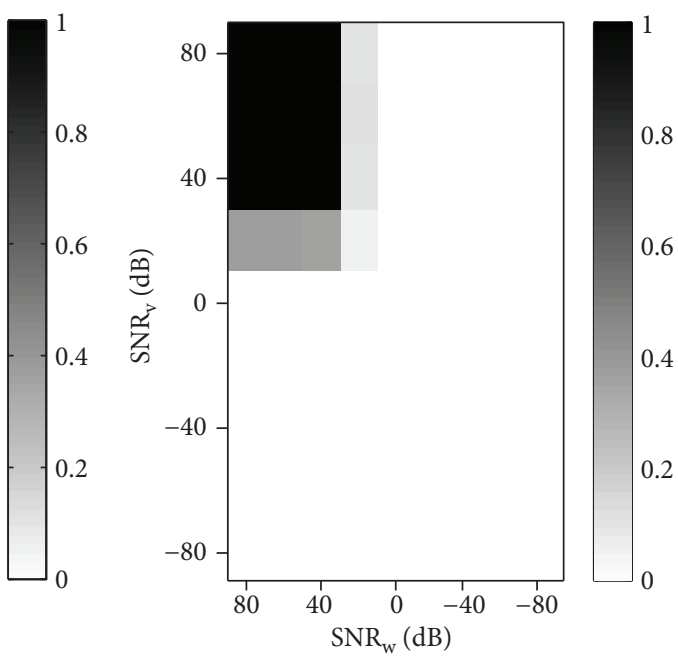

(b)

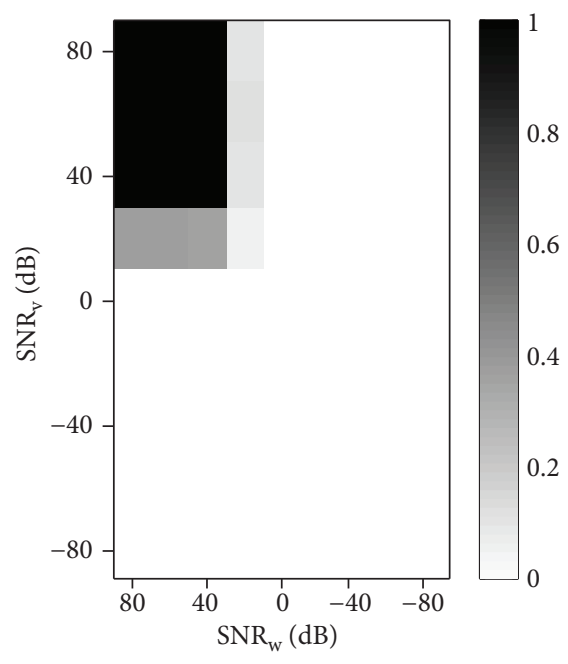

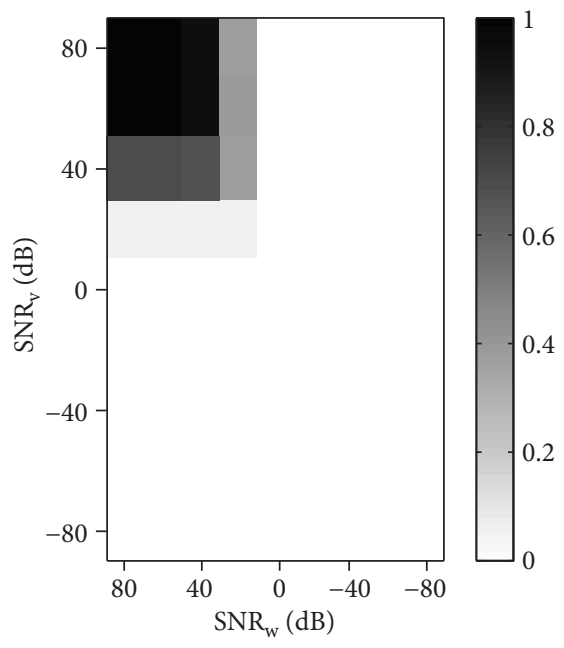

(c)

Figure 7: $P_{\text {Per }}$ of unlabeled GLRT with known $\theta$ detector versus $\operatorname{SNR}_{w}$ and $\operatorname{SNR}_{v}$ with $\left(\mu_{h}, \sigma_{h}^{2}\right)=(1,1)(\mathrm{a}),(0,1)(\mathrm{b}),(1,0)(\mathrm{c})$.

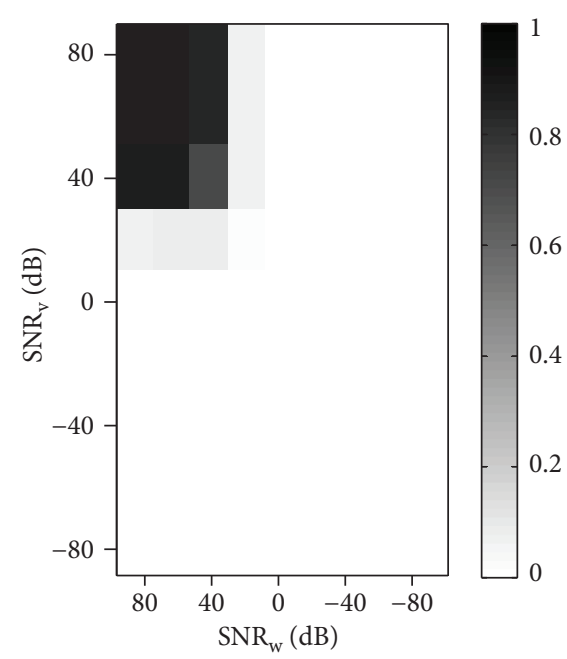

(a)

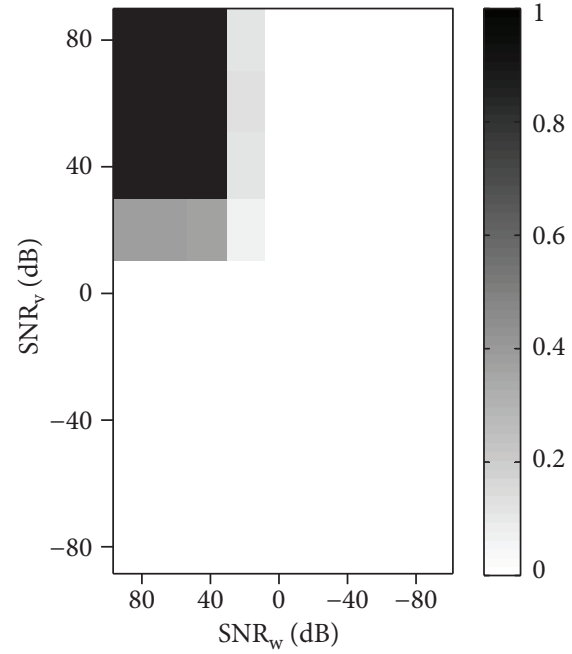

(b)

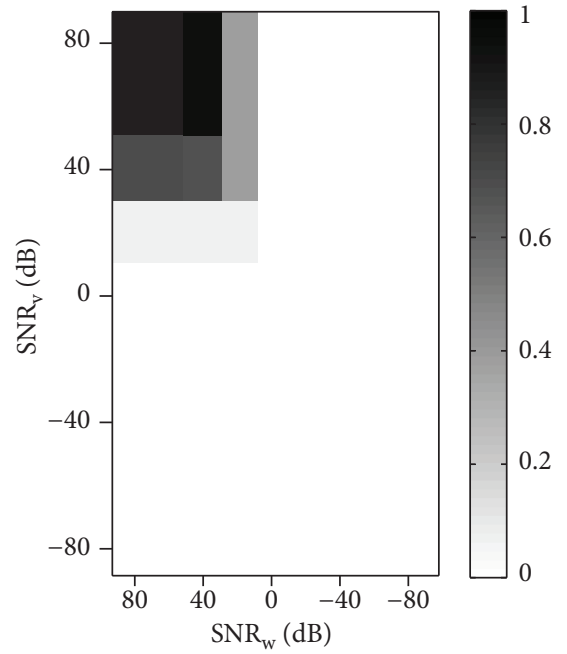

(c)

FIGURE 8: $P_{\text {Per }}$ of unlabeled GLRT with unknown $\theta$ detector versus $\operatorname{SNR}_{w}$ and $\operatorname{SNR}_{v}$ with $\left(\mu_{h}, \sigma_{h}^{2}\right)=(1,1)(\mathrm{a}),(0,1)(\mathrm{b}),(1,0)(\mathrm{c})$.

\section{3. $P_{D}$ versus $N$}

4.3.1. High Noise Level. In the third subsection, the detection performances versus the number of sensors $N$ for various channel coefficients are plotted in Figure 5 in the scenario of high noise level, where $K=5$ and $P_{\mathrm{FA}}=0.05, \sigma_{w}^{2}=10, \sigma_{v}^{2}=$ 10 , respectively, for all cases. As can be seen from Figure 5, the overall tendency is that $P_{\mathrm{D}}$ keeps rising with the increase of $N$, but the curves are not strictly monotonous when $\sigma_{v}^{2}$ is large. In particular, the detection probabilities of the energy detector occasionally decrease in subgraph (d) with the increase of $N$. One reasonable interpretation is that although larger sensor network can provide more observations for signal detection, the growing size of the permutation matrix simultaneously brings more uncertainties. These two factors together produce the slight fluctuation.

4.3.2. Low Noise Level. When the noise level is low, i.e., $\sigma_{w}^{2}=$ $2, \sigma_{v}^{2}=2$, the conclusions from Figure 6 are basically the same as those of the high noise level. But the detection probability $P_{\mathrm{D}}$ reaches 1 more quickly and the curves are more smooth and monotonous.

4.4. $P_{P e r}$ versus $S N R$. In this subsection, the probability of successfully recovering the permutation matrix $\Pi$ versus the $\mathrm{SNR}_{w}$ and $\mathrm{SNR}_{v}$ is plotted in Figure 7 of the unlabeled GLRT 
with known $\theta$ detector and in Figure 8 of the unlabeled GLRT with unknown $\theta$ detector, where the number of sensors $N$ and the signals $K$ is set to be 20 and 5, separately. For $\sigma_{h}^{2}=0$, it can be seen that distinguishing the permutation matrix is meaningless and the results are not plotted. The probability is closer to 1 if the color of the region is closer to black. What we can conclude from Figure 7 and Figure 8 is that, for both detectors, only in the very high-SNR scenarios, the probability is 1 . As the SNR decreases, the recovery probability will quickly reach 0 , implying the transition phase is very sharp. Furthermore, both $\mathrm{SNR}_{w}$ and $\mathrm{SNR}_{v}$ in the previous numerical experiments are less than $-10 \mathrm{~dB}$, which means that the permutation matrix is not recovered correctly. This result also demonstrates that detecting the signal is much easier than recovering the permutation matrix.

\section{Conclusion}

In this paper, distributed constant level detection based on unlabeled network observations is studied. Detection statistics are established for unlabeled and labeled GLRT, mean detector, and energy detector. Theoretical analyses of the various detectors are provided, and their performances are evaluated by performing MC simulations. Numerical results demonstrate that behaviour of the proposed detectors versus the numbers of sensors and the observation time are basically positive related. The unlabeled GLRT detector often performs better than the energy detector. Meanwhile, the performance degradation of the unlabeled GLRT detector is small, comparable to that of the labeled detector.

\section{Data Availability}

The data used to support the findings of this study are available from the corresponding author upon request.

\section{Conflicts of Interest}

The authors declare that they have no conflicts of interest.

\section{References}

[1] J. N. Tsitsiklis, "Decentralized detection," Advances in Statistical Signal Processing, vol. 2, pp. 297-344, 1989.

[2] R. Viswanathan and P. K. Varshney, "Distributed detection with multiple sensors: part I-fundamentals," Proceedings of the IEEE, vol. 85, no. 1, pp. 54-63, 1997.

[3] R. S. Blum, S. A. Kassam, and H.V. Poor, "Distributed detection with multiple sensors: part II-advanced topics," Proceedings of the IEEE, vol. 85, no. 1, pp. 64-79, 1997.

[4] J. F. Chamberland and V.V. Veeravalli, "Decentralized detection in sensor networks," IEEE Transactions on Signal Processing, vol. 51, no. 2, pp. 407-416, 2003.

[5] A. Ribeiro and G. B. Giannakis, "Bandwidth-constrained distributed estimation for wireless sensor networks-part I: Gaussian case," IEEE Transactions on Signal Processing, vol. 54, no. 3, pp. 1131-1143, 2006.

[6] "Bandwidth-constrained distributed estimation for wireless sensor networks-part I: Gaussian case," IEEE Transactions on Signal Processing, vol. 54, no. 3, pp. 1131-1143, 2006.

[7] J. Zhu, X. Wang, X. Lin, and Y. Gu, "Maximum likelihood estimation from sign measurements with sensing matrix perturbation," IEEE Transactions on Signal Processing, vol. 62, pp. 3741-3753, 2014.

[8] J. Zhu, X. Lin, R. S. Blum, and Y. Gu, "Parameter estimation from quantized observations in multiplicative noise environments," IEEE Transactions on Signal Processing, vol. 63, no. 15, pp. 4037-4050, 2015.

[9] J. Zhu, R. S. Blum, X. Lin, and Y. Gu, "Robust transmit beamforming for parameter estimation using distributed sensors," IEEE commun. lett., vol. 20, no. 7, pp. 1329-1332, 2016.

[10] G. Wang, J. Zhu, and Z. Xu, "Asymptotically optimal onebit quantizer design for weak-signal detection in generalized Gaussian noise and lossy binary communication channel," Signal Processing, vol. 154, pp. 207-216, 2018.

[11] Y. Pan, F. Duan, F. Chapeau-Blondeau, and D. Abbott, "Noise enhancement in robust estimation of location," IEEE Transactions on Signal Processing, vol. 66, no. 8, pp. 1953-1966, 2018.

[12] J. Zhu, L. Han, R. S. Blum, and Z. Xu, "On the analysis of the Fisher information of a perturbed linear model after random compression," IEEE Signal Processing Letters, vol. 25, no. 1, pp. 100-104, 2018.

[13] J. Zhu, Q. Zhang, X. Meng, and Z. Xu, "Vector approximate message passing algorithm for structured perturbed sensing matrix," http://arxiv.org/abs/1808.08579.

[14] J. Zhu and X. Lin, Sparse estimation from sign measurements with general sensing matrix perturbation, International Conference on Digital Signal Processing, Hong Kong, pp. 48-53, 2014.

[15] A. Sani and A. Vosoughi, "On distributed linear estimation with observation model uncertainties," IEEE Transactions on Signal Processing, vol. 66, no. 12, pp. 3212-3227, 2018.

[16] V. Emiya, A. Bonnefoy, R. Gribonval, and L. Daudet, “'Compressed sensing with unknown sensor permutation"," ICASSP, pp. 1040-1044, 2014.

[17] J. Unnikrishnan, S. Haghighatshoar, and M. Vetterli, "Unlabeled sensing with random linear measurements"," Allerton Conference, pp. 786-793, 2015.

[18] A. Pananjady and M. J. Wainwright, Courtade, Linear regression with an unknown permutation: Statistical and Computational Limits, to appear in IEEE Transactions Information Theory, 2017.

[19] S. Haghighatshoar and G. Caire, "Signal recovery from unlabeled samples," IEEE Transactions on Signal Processing, vol. 66, no. 5, pp. 1242-1257, 2018.

[20] J. Zhu, H. Cao, C. Song, and Z. Xu, "Parameter estimation via unlabeled sensing using distributed sensors," IEEE Communications Letters, vol. 21, no. 10, pp. 2130-2133, 2017.

[21] Z. Liu and J. Zhu, "Signal detection from unlabeled ordered samples," in 2431, IEEE Communications Letters, 22, p. 12.

[22] G. Wang, J. Zhu, R.S. Blum et al., "Signal amplitude estimation and detection from unlabeled binary quantized samples," IEEE Transactions on Signal Processing, vol. 66, no. 16, pp. 4291-4303, 2018.

[23] G. Wang, J. Zhu, R. S. Blum et al., "Signal amplitude estimation and detection from unlabeled binary quantized samples," IEEE Transactions on Signal Processing, vol. 66, no. 16, pp. 4291-4303, 2018.

[24] W. S. Robinson, "A method for chronologically ordering archaeological deposits," American Antiquity, vol. 16, no. 4, pp. 293-301, 1951.

[25] A. V. Balakrishnan, "On the problem of time jitter in sampling," IRE Transactions Information Theory, vol. 8, no. 3, pp. 226-236, 1962. 
[26] A. B. Poore and S. Gadaleta, "Some assignment problems arising from multiple target tracking," Mathematical and Computer Modelling, vol. 43, no. 9, pp. 1074-1091, 2006.

[27] S. Marano, V. Matta, P. Willett, P. Braca, and R. S. Blum, "Hypothesis testing in the presence of Maxwell's daemon: signal detection by unlabeled observations," ICASSP, pp. 3286-3290, 2017.

[28] A. Pananjady, M. J. Wainwright, and T. A. Courtade, "Denoising Linear Models with Permuted Data," in 2017 IEEE ISIT, 2017. 


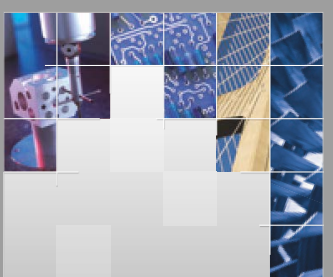

\section{Enfincering}
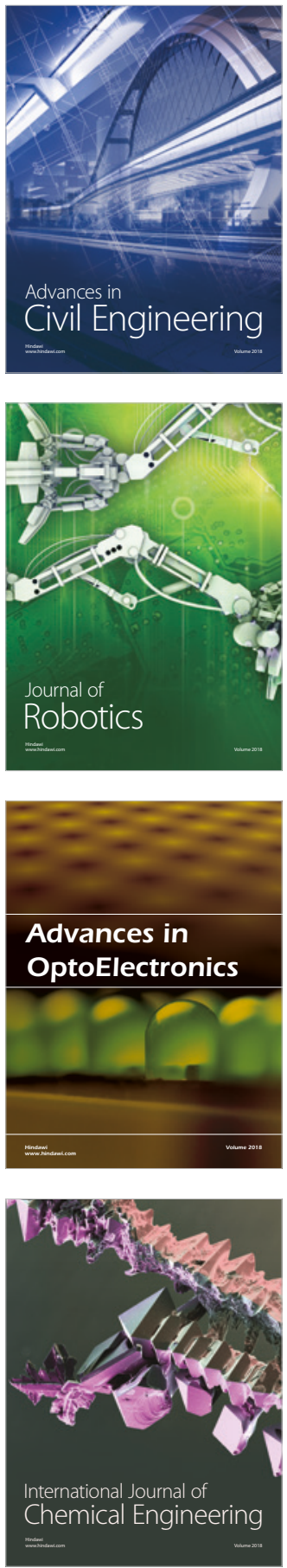

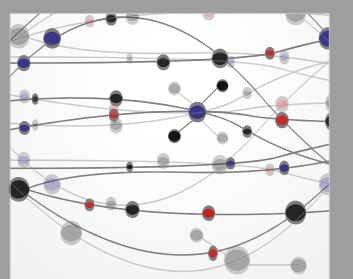

\section{Rotating \\ Machinery}

The Scientific World Journal

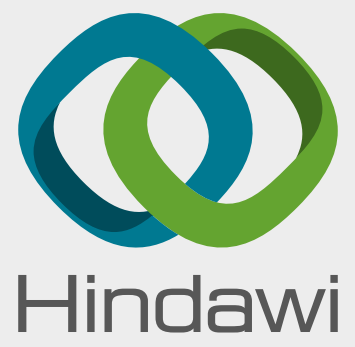

Submit your manuscripts at

www.hindawi.com
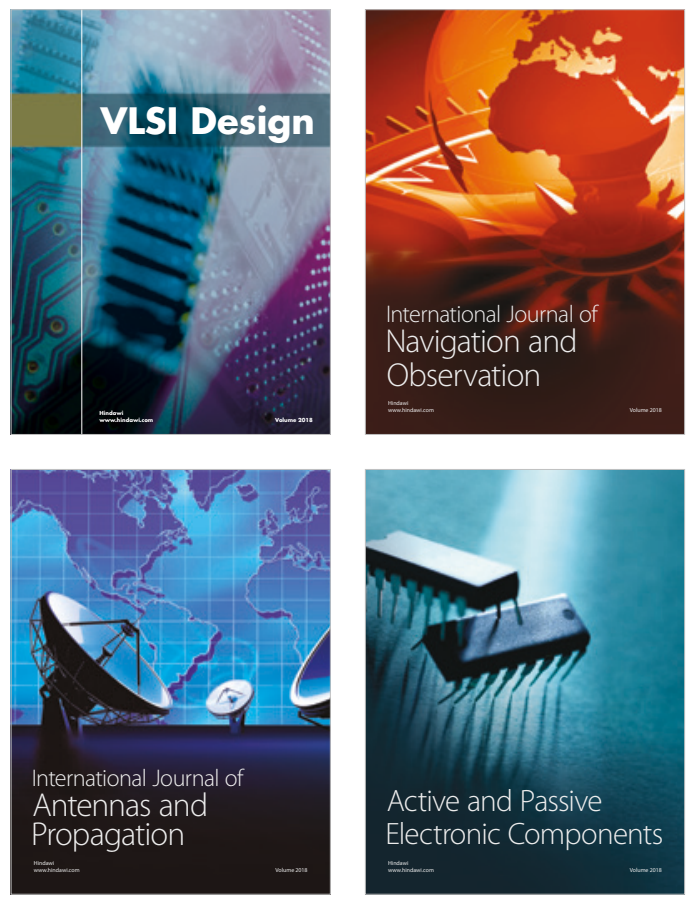
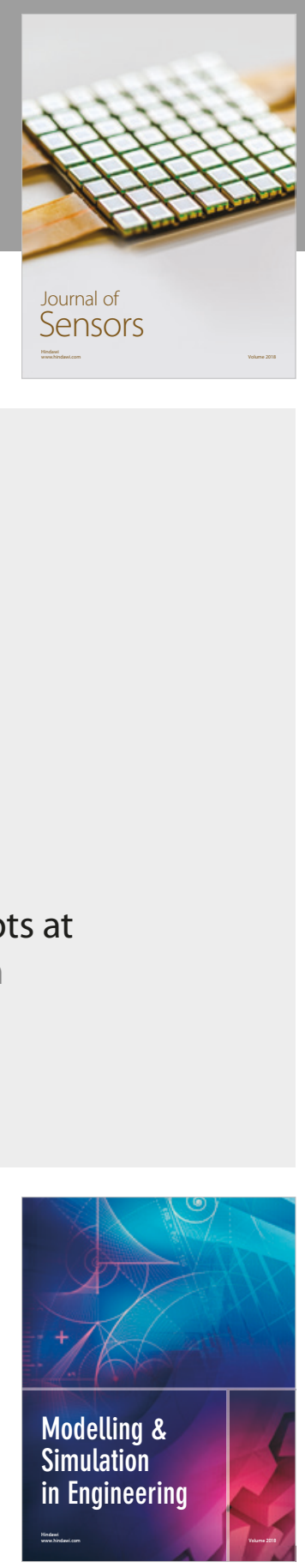

\section{Advances \\ Multimedia}
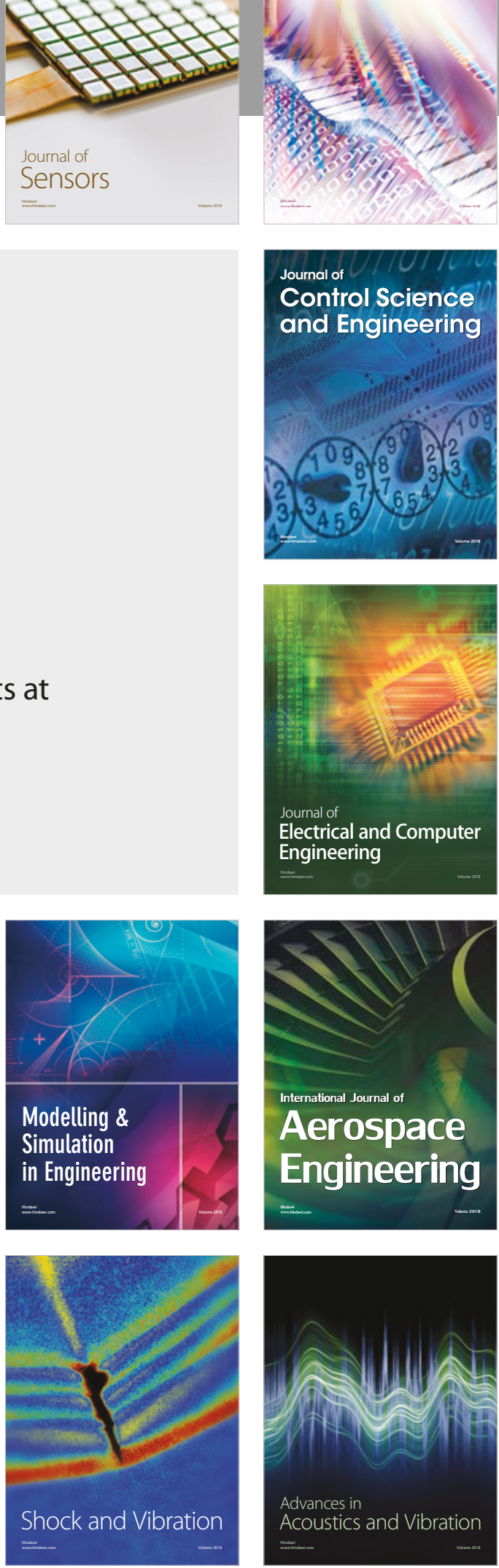